\title{
A SUPERLATIVE INDICATOR FOR THE LUENBERGER-HICKS-MOORSTEEN PRODUCTIVITY INDICATOR: THEORY AND APPLICATION
}

\author{
FREDERIC ANG AND PIETER JAN KERSTENS
}

\begin{abstract}
Consisting of the difference between an output indicator and an input indicator, the Luenberger-Hicks-Moorsteen (LHM) productivity indicator allows straightforward interpretation. However, its computation requires estimating distance functions that are inherently unknown. This paper shows that a computationally simple Bennet indicator is a superlative indicator for the LHM indicator when one can assume profit-maximizing behavior and the input and output directional distance functions can be represented up to the second order by a quadratic functional form. We also show that the Luenberger- and LHM-approximating Bennet indicators coincide for an appropriate choice of directional vectors. Focusing on a large sample of Italian food and beverages companies for the years 1995 - 2007, we empirically investigate the extent to which this theoretical equivalence translates into similar estimates. We find that the Bennet indicator is a close empirical alternative to the LHM indicator for the sample. Keywords: Productivity and competitiveness, Bennet, Luenberger-Hicks-Moorsteen, superlative, Italian food and beverages sector.
\end{abstract}

JEL classification: C43, D21, D22, D24.

Business Economics Group, Wageningen University, P.O. Box 8130, 6700 EW Wageningen, The Netherlands

Department of Food and Resource Economics (IFRO), University of Copenhagen, RoLighedsvej 25, DK-1958 Frederiksberg C, Denmark

Date: February 17, 2020.

We thank Bert Balk and Hideyuki Mizobuchi for comments on an earlier working paper without application (Ang and Kerstens, 2017b). The authors gratefully acknowledge four anonymous referees for their valuable comments and suggestions. Both authors have contributed equally to this paper. Email pjk@ifro.ku.dk for correspondence. The usual disclaimer applies. 


\section{INTRODUCTION}

Productivity analysis is an essential tool to benchmark economic performance. Following Lovell (2016), there are two approaches to productivity measurement. The theoretical approach to productivity measurement entails estimation of distance functions. Not requiring any estimation of distance functions, the empirical approach employs a computationally simple empirical index number formula of prices and quantities of inputs and outputs. The production technology underlying distance functions is inherently unknown and their computation is often complicated, while empirical index numbers are easily computed. Therefore, finding empirical index numbers that approximate distancefunction-based productivity measures is of practical interest to the empirical analyst. An important body of literature initiated by Diewert (1976) seeks to find "superlative" index numbers for which the approximation holds under the assumption of (i) economic optimizing behavior and (ii) a technology that can be represented up to the second order by a flexible functional form.

No superlative index number is currently known for the Luenberger-Hicks-Moorsteen (LHM) productivity indicator developed by Briec and Kerstens (2004). Consisting of output and input directional distance functions, the LHM indicator follows the theoretical approach. It has several attractive theoretical properties. First, it is difference-based. Ratio-based "indexes" can be undefined when zeros occur in the numerator or denominator and are not translation-invariant. Difference-based "indicators" avoid these drawbacks altogether (Balk et al., 2003). Second, the LHM indicator is additively complete, which means that it consists of the difference between an output indicator and an input indicator (O'Donnell, 2012). Third, requiring only quantity information, the LHM indicator can be computed even if there is no price information. Its straightforward interpretation and desirable theoretical properties make the LHM indicator suitable for empirical application (e.g. Ang and Kerstens, 2017a; Kerstens et al., 2018; MolinosSenante et al., 2019; Sala-Garrido et al., 2018; Seufert et al., 2017; Shen et al., 2019). Nonetheless, the computation of inherently unknown distance functions complicates the practical use of the LHM indicator. The current paper addresses this issue by revealing a superlative indicator for the LHM indicator that can be applied when data on quantities as well as prices of inputs and outputs are available.

Our superlative indicator complements several superlative index number formulae from the literature. Caves et al. (1982) show that the Törnqvist input (output) index is a superlative index for the input (output) Malmquist index when there is cost-minimizing (revenue-maximizing) behavior, and the input (output) distance functions can be represented up to the second order by a translog functional form. This result also holds for the Fisher ideal index (Diewert, 1992). Diewert and Fox (2010) claim that the equivalence 
between the Törnqvist input (output) index and Malmquist input (output) index does not rely on any assumption about returns to scale. Following this reasoning, they argue that the Törnqvist index, being the ratio of the Törnqvist output index to the Törnqvist input index, coincides with Bjurek (1996)'s Hicks-Moorsteen index, being the ratio of the Malmquist output index to the Malmquist input index, without any assumptions about returns to scale under the same theoretical conditions. Mizobuchi (2017) shows that constant-returns-to-scale are essential for the equivalence between the Törnqvist index and both the Malmquist index and Hicks-Moorsteen index. Furthermore, he illustrates that this condition can be loosened to $\alpha$-returns-to-scale for the Hicks-Moorsteen index, but not for the Malmquist index. Balk (1998) and Chambers $(1996,2002)$ show that the Bennet indicator is a superlative indicator for Chambers et al. (1996)'s Luenberger indicator when there is profit-maximizing behavior, and the directional distance functions can be represented up to the second order by a quadratic functional form with time-invariant second-order coefficients.

The contributions of this paper are threefold. First, we show that the Bennet indicator coincides with the LHM indicator when there is profit-maximizing behavior, and the input and output directional distance functions can be represented up to the second order by a quadratic functional form with time-invariant second-order coefficients. The Bennet indicator is thus a superlative indicator for Luenberger as well as LHM indicators (albeit for a different price normalization). Its straightforward computation, differencebased structure and close relationship with profit measures (Balk, 1998; Grifell-Tatjé and Lovell, 1999) make the Bennet indicator an attractive accountancy-based alternative for management control purposes. Second, we show the theoretical conditions under which the Luenberger-approximating Bennet indicator is equivalent to the LHM-approximating Bennet indicator. Third, focusing on a large sample of Italian food and beverages companies for the years $1995-2007$, we empirically investigate the extent to which this theoretical equivalence translates into similar estimates. The food and beverages industry is the largest manufacturing sector in the European Union. The Italian food and beverages industry has a value added of $12.6 \%$ of the total value added of the EU-28 in 2012, which makes it the third largest contributor among the EU member states (Eurostat, 2018).

The remainder of this paper is structured as follows. The next section introduces necessary notation and defines Luenberger and Bennet indicators. We then define the LHM indicator and present the theoretical equivalence between the LHM and Bennet indicator. This is followed by the empirical application to the Italian food and beverages companies. The final section concludes. 


\section{Linking the Luenberger Productivity indicator to the Bennet Profit} INDICATOR

This section sets the stage for our main result by introducing necessary notation and definitions of the Luenberger indicator and the Bennet cost, revenue and profit indicators. It also reminds the reader of the result of Balk (1998) and Chambers (1996, 2002), which links the Bennet profit indicator as a superlative approximation of the Luenberger indicator.

2.1. The Luenberger productivity indicator. Let $\mathbf{x}_{t} \in \mathbb{R}_{+}^{n}$ be the inputs that are used to produce outputs $\mathbf{y}_{t} \in \mathbb{R}_{+}^{m}$. We define the production possibility set as:

$$
\mathcal{T}_{t}=\left\{\left(\mathbf{x}_{t}, \mathbf{y}_{t}\right) \in \mathbb{R}_{+}^{n+m} \mid \mathbf{x}_{t} \text { can produce } \mathbf{y}_{t}\right\} \text {. }
$$

We make the following assumptions on the production possibility set (Chambers, 2002):

Axiom 1 (Closedness). $\mathcal{T}_{t}$ is closed.

Axiom 2 (Free disposability of inputs and outputs). if $\left(\mathbf{x}_{t}^{\prime},-\mathbf{y}_{t}^{\prime}\right) \geq\left(\mathbf{x}_{t},-\mathbf{y}_{t}\right)$ then $\left(\mathbf{x}_{t}, \mathbf{y}_{t}\right) \in \mathcal{T}_{t} \Rightarrow\left(\mathbf{x}_{t}^{\prime}, \mathbf{y}_{t}^{\prime}\right) \in \mathcal{T}_{t}$.

Axiom 3 (Inaction). Inaction is possible: $\left(\mathbf{0}^{n}, \mathbf{0}^{m}\right) \in \mathcal{T}_{t}$.

The directional distance function was first introduced in a production context by Chambers et al. (1996). We denote the time-related directional distance function for $(a, b) \in\{t, t+1\} \times\{t, t+1\}:$

$$
D_{b}\left(\mathbf{x}_{a}, \mathbf{y}_{a} ; \mathbf{g}\right)=\sup \left\{\beta \in \mathbb{R}:\left(\mathbf{x}_{a}-\beta \mathbf{g}^{i}, \mathbf{y}_{a}+\beta \mathbf{g}^{o}\right) \in \mathcal{T}_{b}\right\},
$$

if $\left(\mathbf{x}_{a}-\beta \mathbf{g}^{i}, \mathbf{y}_{a}+\beta \mathbf{g}^{o}\right) \in \mathcal{T}_{b}$ for some $\beta$ and $D_{b}\left(\mathbf{x}_{a}, \mathbf{y}_{a} ; \mathbf{g}\right)=-\infty$ otherwise. Here, $\mathbf{g}=\left(\mathbf{g}^{i}, \mathbf{g}^{o}\right)$ represents the directional vector.

Chambers (2002) defines the Luenberger productivity indicator as:

$$
\begin{aligned}
& L_{t, t+1}\left(\mathbf{x}_{t}, \mathbf{y}_{t}, \mathbf{x}_{t+1}, \mathbf{y}_{t+1} ; \mathbf{g}\right) \\
& \quad=\frac{1}{2}\left[\left(D_{t}\left(\mathbf{x}_{t}, \mathbf{y}_{t} ; \mathbf{g}\right)-D_{t}\left(\mathbf{x}_{t+1}, \mathbf{y}_{t+1} ; \mathbf{g}\right)\right)\right. \\
& \left.\quad+\left(D_{t+1}\left(\mathbf{x}_{t}, \mathbf{y}_{t} ; \mathbf{g}\right)-D_{t+1}\left(\mathbf{x}_{t+1}, \mathbf{y}_{t+1} ; \mathbf{g}\right)\right)\right] .
\end{aligned}
$$

It can be decomposed in technical change and technical inefficiency change (Chambers et al., 1996), but the exact contribution of output and input change cannot be determined. This is because, in general, $\mathbf{g}=\left(\mathbf{g}^{i}, \mathbf{g}^{o}\right)>\left(\mathbf{0}^{n}, \mathbf{0}^{m}\right)$ and inputs are contracted simultaneously as outputs are expanded in the directional distance functions (Ang and 
Kerstens, 2017a). Hence, it is not "additively complete" (O'Donnell, 2012). Furthermore, unlike the Luenberger-Hicks-Moorsteen (Briec and Kerstens, 2011), the Luenberger productivity indicator is not "determinate" in that it can be undefined (Briec and Kerstens, 2009). ${ }^{1}$

Furthermore, Chambers (2002) defines the output Luenberger productivity indicator as:

$$
L O_{t, t+1}\left(\mathbf{x}_{t}, \mathbf{y}_{t}, \mathbf{x}_{t+1}, \mathbf{y}_{t+1} ; \mathbf{g}^{o}\right)=\frac{1}{2}\left[L O_{t}+L O_{t+1}\right]
$$

where the base period $t$ output indicator is defined as:

$$
L O_{t}\left(\mathbf{x}_{t}, \mathbf{y}_{t}, \mathbf{y}_{t+1} ; \mathbf{g}^{o}\right)=D_{t}\left(\mathbf{x}_{t}, \mathbf{y}_{t} ;\left(\mathbf{0}^{n}, \mathbf{g}^{o}\right)\right)-D_{t}\left(\mathbf{x}_{t}, \mathbf{y}_{t+1} ;\left(\mathbf{0}^{n}, \mathbf{g}^{o}\right)\right),
$$

and the base period $t+1$ output indicator:

$$
L O_{t+1}\left(\mathbf{x}_{t+1}, \mathbf{y}_{t+1}, \mathbf{y}_{t} ; \mathbf{g}^{o}\right)=D_{t+1}\left(\mathbf{x}_{t+1}, \mathbf{y}_{t} ;\left(\mathbf{0}^{n}, \mathbf{g}^{o}\right)\right)-D_{t+1}\left(\mathbf{x}_{t+1}, \mathbf{y}_{t+1} ;\left(\mathbf{0}^{n}, \mathbf{g}^{o}\right)\right)
$$

The input Luenberger productivity indicator is:

$$
L I_{t, t+1}\left(\mathbf{x}_{t}, \mathbf{y}_{t}, \mathbf{x}_{t+1}, \mathbf{y}_{t+1} ; \mathbf{g}^{i}\right)=\frac{1}{2}\left[L I_{t}+L I_{t+1}\right]
$$

where the base period $t$ input indicator is defined as:

$$
L I_{t}\left(\mathbf{x}_{t}, \mathbf{x}_{t+1}, \mathbf{y}_{t} ; \mathbf{g}^{i}\right)=D_{t}\left(\mathbf{x}_{t}, \mathbf{y}_{t} ;\left(\mathbf{g}^{i}, \mathbf{0}^{m}\right)\right)-D_{t}\left(\mathbf{x}_{t+1}, \mathbf{y}_{t} ;\left(\mathbf{g}^{i}, \mathbf{0}^{m}\right)\right)
$$

and the base period $t+1$ input indicator as:

$$
L I_{t+1}\left(\mathbf{x}_{t}, \mathbf{x}_{t+1}, \mathbf{y}_{t+1} ; \mathbf{g}^{i}\right)=D_{t+1}\left(\mathbf{x}_{t}, \mathbf{y}_{t+1} ;\left(\mathbf{g}^{i}, \mathbf{0}^{m}\right)\right)-D_{t+1}\left(\mathbf{x}_{t+1}, \mathbf{y}_{t+1} ;\left(\mathbf{g}^{i}, \mathbf{0}^{m}\right)\right)
$$

The output (input) Luenberger productivity indicator $L O_{t, t+1}(\cdot)\left(L I_{t, t+1}(\cdot)\right)$ measures productivity solely in the output (input) directions. Thus, a combination of both $L O_{t, t+1}(\cdot)$ and $L I_{t, t+1}(\cdot)$ is "additively complete". This is Briec and Kerstens (2004)'s Luenberger-Hicks-Moorsteen productivity indicator (see Section 3 infra), with timeinvariant directional vectors.

2.2. Bennet indicators. The preceding productivity measures have the advantage that they can be computed in the absence of price data, but their major drawback is that they require the approximation of the technology set and estimation of distance functions. This makes them somewhat harder to compute. We now focus our attention to productivity measures that are easy to compute using price data and which do not require estimation of distance functions.

\footnotetext{
${ }^{1}$ Determinateness of the indicator does not necessarily carry over to its components. This occurs for example in the empirical application of Ang and Kerstens (2017a), where the technical change component is undefined for one of the observations.
} 
Consider the following directional distance function at time $h$ :

$$
\begin{aligned}
D_{h}\left(\mathbf{x}, \mathbf{y} ;\left(\mathbf{g}^{i}, \mathbf{g}^{o}\right)\right) & =a_{h}^{0}+\sum_{u=1}^{n} a_{h}^{u} x^{u}+\sum_{k=1}^{m} b_{h}^{k} y^{k}+\frac{1}{2} \sum_{u=1}^{n} \sum_{v=1}^{n} \alpha_{h}^{u v} x^{u} x^{v} \\
& +\frac{1}{2} \sum_{k=1}^{m} \sum_{l=1}^{m} \beta_{h}^{k l} y^{k} y^{l}+\sum_{u=1}^{n} \sum_{k=1}^{m} \gamma_{h}^{u k} x^{u} y^{k}
\end{aligned}
$$

with the restrictions

$$
\begin{array}{ll}
\alpha_{h}^{u v}=\alpha_{h}^{v u}, \beta_{h}^{k l}=\beta_{h}^{l k}, & \\
\sum_{k=1}^{m} b_{h}^{k} g_{k}^{o}-\sum_{u=1}^{n} a_{h}^{u} g_{u}^{i}=-1 ; & \\
\sum_{k=1}^{m} \gamma_{h}^{u k} g_{k}^{o}-\sum_{v=1}^{n} \alpha_{h}^{u v} g_{v}^{i}=0, & k=1, \ldots, n ; \\
\sum_{l=1}^{m} \beta_{h}^{k l} g_{l}^{o}-\sum_{u=1}^{n} \gamma_{h}^{u k} g_{u}^{i}=0, &
\end{array}
$$

We refer to Hudgins and Primont (2007) for a detailed derivation and explanation of these restrictions. The directional distance function is a measure of technical inefficiency, which measures the ability to simultaneously expand output production and contract input use, in terms of the directional vector $\mathbf{g}$. The output (input) directional distance function is defined by setting $\mathbf{g}^{i(o)}=\mathbf{0}^{n(m)}$.

This directional distance function has a quadratic functional form (5a). Following Chambers (2002, Lemma 3), among others, we interpret the equation (5a) as "flexible" in Diewert (1976)'s sense, as it provides a second order approximation over the technology set $\mathcal{T}_{h}$ at an arbitrary point $\left(\mathbf{x}^{*}, \mathbf{y}^{*}\right)$. We thus follow the interpretation of Balk (1998) and Chambers (2002) that $\left(\mathbf{g}^{i}, \mathbf{g}^{o}\right)$ is given. ${ }^{2}$

Can both $D_{h}\left(\mathbf{x}, \mathbf{y} ;\left(\mathbf{g}^{i}, \mathbf{0}^{m}\right)\right)$ and $D_{h}\left(\mathbf{x}, \mathbf{y} ;\left(\mathbf{0}^{n}, \mathbf{g}^{o}\right)\right)$ simultaneously have the quadratic functional form (5)? Not necessarily so ${ }^{3}$, but under fairly loose conditions this holds. Consider the following general relationship between $D_{h}\left(\mathbf{x}, \mathbf{y} ;\left(\mathbf{g}^{i}, \mathbf{0}^{m}\right)\right)$ and $D_{h}\left(\mathbf{x}, \mathbf{y} ;\left(\mathbf{0}^{n}, \mathbf{g}^{o}\right)\right)$ (see e.g., Briec et al. (2006, p. 293)):

$$
D_{h}\left(\mathbf{x}, \mathbf{y} ;\left(\mathbf{0}^{n}, \mathbf{g}^{o}\right)\right)=\sup \left\{\beta: D_{h}\left(\mathbf{x}, \mathbf{y}+\beta \mathbf{g}^{o} ;\left(\mathbf{g}^{i}, \mathbf{0}^{m}\right)\right) \geq 0\right\}
$$

\footnotetext{
${ }^{2}$ As pointed out by an anonymous reviewer, it does not provide a second order approximation at an arbitrary point $\left(\mathbf{x}^{*}, \mathbf{y}^{*}, \mathbf{g}^{i *}, \mathbf{g}^{o *}\right)$.

${ }^{3}$ We are grateful to an anonymous reviewer for pointing this out to us.
} 
Now suppose that $D_{h}\left(\mathbf{x}, \mathbf{y} ;\left(\mathbf{g}^{i}, \mathbf{0}^{m}\right)\right)$ has the quadratic functional form. Solving for $D_{h}\left(\mathbf{x}, \mathbf{y} ;\left(\mathbf{0}^{n}, \mathbf{g}^{o}\right)\right)$ using $(6)$, one sees that $D_{h}\left(\mathbf{x}, \mathbf{y} ;\left(\mathbf{0}^{n}, \mathbf{g}^{o}\right)\right)$ does not necessarily have the same quadratic functional form.

The following Lemma imposes sufficient conditions under which both $D_{h}\left(\mathbf{x}, \mathbf{y} ;\left(\mathbf{g}^{i}, \mathbf{0}^{m}\right)\right)$ and $D_{h}\left(\mathbf{x}, \mathbf{y} ;\left(\mathbf{0}^{n}, \mathbf{g}^{o}\right)\right)$ simultaneously have the quadratic functional form.

Lemma 1 (Affine Transformation Condition). Let the output directional distance function be an affine transformation of the input directional distance function: $D_{h}\left(\mathbf{x}, \mathbf{y} ;\left(\mathbf{0}^{n}, \mathbf{g}^{o}\right)\right)=$ $\zeta D_{h}\left(\mathbf{x}, \mathbf{y} ;\left(\mathbf{g}^{i}, \mathbf{0}^{m}\right)\right)+\eta$ with $\zeta \in \mathbb{R}_{0}, \eta \in \mathbb{R}$. Then $D_{h}\left(\mathbf{x}, \mathbf{y} ;\left(\mathbf{0}^{n}, \mathbf{g}^{o}\right)\right)$ has the quadratic functional form (5) if and only if $D_{h}\left(\mathbf{x}, \mathbf{y} ;\left(\mathbf{g}^{i}, \mathbf{0}^{m}\right)\right)$ has the quadratic functional form (5).

Proof. Assume that the relationship $D_{h}\left(\mathbf{x}, \mathbf{y} ;\left(\mathbf{0}^{n}, \mathbf{g}^{o}\right)\right)=\zeta D_{h}\left(\mathbf{x}, \mathbf{y} ;\left(\mathbf{g}^{i}, \mathbf{0}^{m}\right)\right)+\eta$ with some $\zeta \in \mathbb{R}_{0}, \eta \in \mathbb{R}$ holds. The following holds:

$$
\begin{aligned}
D_{h}\left(\mathbf{x}, \mathbf{y} ;\left(\mathbf{0}^{n}, \mathbf{g}^{o}\right)\right) & =\zeta D_{h}\left(\mathbf{x}, \mathbf{y} ;\left(\mathbf{g}^{i}, \mathbf{0}^{m}\right)\right)+\eta \\
& =D_{h}\left(\mathbf{x}, \mathbf{y} ;\left(\frac{\mathbf{g}^{i}}{\zeta}, \mathbf{0}^{m}\right)\right)+\eta .
\end{aligned}
$$

$\Rightarrow$ Suppose that $D_{h}\left(\mathbf{x}, \mathbf{y} ;\left(\mathbf{g}^{i}, \mathbf{0}^{m}\right)\right)$ has the quadratic functional form (5). One sees immediately that $D_{h}\left(\mathbf{x}, \mathbf{y} ;\left(\mathbf{0}^{n}, \mathbf{g}^{o}\right)\right)$ also has the quadratic functional form (5a), because the latter intercept can be redefined by $\tilde{a}_{h}^{0} \equiv a_{h}^{0}+\eta$. This establishes that both have the quadratic functional form (5a).

Next, we must show that the restrictions (5b)-(5e) on the parameters of the quadratic functional form do not render the feasible parameter set for $D_{h}\left(\mathbf{x}, \mathbf{y} ;\left(\mathbf{0}^{n}, \mathbf{g}^{o}\right)\right)$ empty. Let $\Theta^{i}\left(\Theta^{o}\right)$ be the feasible parameter set for $D_{h}\left(\mathbf{x}, \mathbf{y} ;\left(\mathbf{g}^{i}, \mathbf{0}^{m}\right)\right)\left(D_{h}\left(\mathbf{x}, \mathbf{y} ;\left(\mathbf{0}^{n}, \mathbf{g}^{o}\right)\right)\right)$ satisfying the restrictions (5b)-(5e). Thus, we must show that $\Theta^{o} \neq \emptyset$ when $\Theta^{i} \neq \emptyset$. By assumption $D_{h}\left(\mathbf{x}, \mathbf{y} ;\left(\mathbf{g}^{i}, \mathbf{0}^{m}\right)\right)$ has the quadratic functional form (5) and thus $\Theta^{i} \neq \emptyset$. Choose any $\theta^{i}=\left(a_{h}^{0}, \mathbf{a}_{h}, \mathbf{b}_{h}, \boldsymbol{\alpha}_{h}, \boldsymbol{\beta}_{h}, \boldsymbol{\gamma}_{h}\right) \in \Theta^{i}$. Next, let $\theta^{o}=\left(\tilde{a}_{h}^{0}, \mathbf{a}_{h}, \tilde{\mathbf{b}}_{h}, \boldsymbol{\alpha}_{h}, \tilde{\boldsymbol{\beta}}_{h}, \tilde{\gamma}_{h}\right)$ where $\tilde{\mathbf{b}}_{h}=$ $\mathbf{b}_{h}, \tilde{\boldsymbol{\beta}}_{h}=\boldsymbol{\beta}_{h}, \tilde{\boldsymbol{\gamma}}_{h}=\boldsymbol{\gamma}_{h}$ with the following modifications to these parameters:

$$
\begin{aligned}
\tilde{b}_{h}^{m}=\frac{-\sum_{k=1}^{m-1} b_{h}^{k} g_{k}^{o}-1}{g_{m}^{o}} & \\
\tilde{\gamma}_{h}^{u m}=-\frac{\sum_{k=1}^{m-1} \gamma_{h}^{u k} g_{k}^{o}}{g_{m}^{o}} & \forall u=1, \ldots, n \\
\tilde{\beta}_{h}^{k k}=-\frac{\sum_{l \in\{1, \ldots, m\} \backslash k} \beta_{h}^{k l} g_{l}^{o}}{g_{k}^{o}} & \forall k=1, \ldots, m
\end{aligned}
$$

By construction these parameters satisfy the restrictions (5b)-(5e). The symmetry conditions (5b) remain satisfied, because only the diagonal elements of $\boldsymbol{\beta}_{h}$ are modified. 
Further, (5b)-(5e) for $\mathbf{g}=\left(\mathbf{0}^{n}, \mathbf{g}^{o}\right)$ put no restrictions on $\mathbf{a}_{h}$ and $\boldsymbol{\alpha}_{h}$ so these can be retained as such. It follows that $\theta^{o} \in \Theta^{o}$ and $\Theta^{o} \neq \emptyset$ as we needed to show.

$\Leftarrow$ Suppose that $D_{h}\left(\mathbf{x}, \mathbf{y} ;\left(\mathbf{0}^{n}, \mathbf{g}^{o}\right)\right)$ has the quadratic functional form (5). Again, it follows immediately that $D_{h}\left(\mathbf{x}, \mathbf{y} ;\left(\mathbf{g}^{i}, \mathbf{0}^{m}\right)\right)$ has the quadratic functional form (5a) by redefining its intercept by $\tilde{a}_{h}^{0} \equiv a_{h}^{0}-\eta$. By an analogous reasoning as before one finds that $\Theta^{i} \neq \emptyset$ when $\Theta^{o} \neq \emptyset$.

The special case $\zeta=1, \eta=0$ implies $D_{h}\left(\mathbf{x}, \mathbf{y} ;\left(\mathbf{0}^{n}, \mathbf{g}^{o}\right)\right)=D_{h}\left(\mathbf{x}, \mathbf{y} ;\left(\mathbf{g}^{i}, \mathbf{0}^{m}\right)\right)$ and occurs in case of a graph translation homothetic technology, which is a difference-based alternative to CRS (see e.g., Chambers (2002, Lemma 2)). We provide a simple test in the empirical application for verifying the plausibility of this affine transformation condition.

The preceding Lemma only establishes sufficient conditions under which both quadratic directional distance functions satisfying (5b)-(5e) exist. It does not establish any connection between the parameters of both directional distance functions under the affine transformation condition. In what follows, we show a characterization of the parameters that complies with the affine transformation condition.

Lemma 2. Let $D_{h}\left(\mathbf{x}, \mathbf{y} ;\left(\mathbf{0}^{n}, \mathbf{g}^{o}\right)\right)=\zeta D_{h}\left(\mathbf{x}, \mathbf{y} ;\left(\mathbf{g}^{i}, \mathbf{0}^{m}\right)\right)+\eta$ with some $\zeta \in \mathbb{R}_{0}, \eta \in \mathbb{R}$ hold. Suppose we have a quadratic $D_{h}\left(\mathbf{x}, \mathbf{y} ;\left(\mathbf{0}^{n}, \mathbf{g}^{o}\right)\right)$ or a quadratic $D_{h}\left(\mathbf{x}, \mathbf{y} ;\left(\mathbf{g}^{i}, \mathbf{0}^{m}\right)\right)$ with parameters $\boldsymbol{\theta}=\left(a_{h}^{0}, \mathbf{a}_{h}, \mathbf{b}_{h}, \boldsymbol{\alpha}_{h}, \boldsymbol{\beta}_{h}, \boldsymbol{\gamma}_{h}\right)$ satisfying the conditions:

$$
\begin{gathered}
\alpha_{h}^{u v}=\alpha_{h}^{v u}, \beta_{h}^{k l}=\beta_{h}^{l k} ; \\
\sum_{k=1}^{m} b_{h}^{k} g_{k}^{o}=-1 ; \\
-\zeta \sum_{u=1}^{n} a_{h}^{u} g_{u}^{i}=-1 ; \\
\sum_{k=1}^{m} \gamma_{h}^{u k} g_{k}^{o}=0, \\
-\zeta \sum_{v=1}^{n} \alpha_{h}^{u v} g_{v}^{i}=0, \\
\sum_{l=1}^{m} \beta_{h}^{k l} g_{l}^{o}=0, \\
-\zeta \sum_{u=1}^{n} \gamma_{h}^{u k} g_{u}^{i}=0,
\end{gathered}
$$

Then both $D_{h}\left(\mathbf{x}, \mathbf{y} ;\left(\mathbf{0}^{n}, \mathbf{g}^{o}\right)\right)$ with parameters $\boldsymbol{\theta}^{o}=\boldsymbol{\theta}$ and $D_{h}\left(\mathbf{x}, \mathbf{y} ;\left(\mathbf{g}^{i}, \mathbf{0}^{m}\right)\right)$ with $\boldsymbol{\theta}^{i}=\frac{\boldsymbol{\theta}}{\zeta}$ have the quadratic functional form (5a) subject to the parameter restrictions (5b)-(5e). 
Proof. The affine transformation holds:

$$
D_{h}\left(\mathbf{x}, \mathbf{y} ;\left(\mathbf{0}^{n}, \mathbf{g}^{o}\right)\right)=\zeta D_{h}\left(\mathbf{x}, \mathbf{y} ;\left(\mathbf{g}^{i}, \mathbf{0}^{m}\right)\right)+\eta .
$$

From Lemma 1, the affine transformation relation and the fact that either $D_{h}\left(\mathbf{x}, \mathbf{y} ;\left(\mathbf{0}^{n}, \mathbf{g}^{o}\right)\right)$ or $D_{h}\left(\mathbf{x}, \mathbf{y} ;\left(\mathbf{g}^{i}, \mathbf{0}^{m}\right)\right)$ is quadratic, it follows that both have the quadratic functional form. The affine transformation means that the parameters of $D_{h}\left(\mathbf{x}, \mathbf{y} ;\left(\mathbf{0}^{n}, \mathbf{g}^{o}\right)\right)$ must also correctly represent $\zeta D_{h}\left(\mathbf{x}, \mathbf{y} ;\left(\mathbf{g}^{i}, \mathbf{0}^{m}\right)\right)$. This means that the parameters simultaneously satisfy (5a)-(5e) for $\mathbf{g}=\left(\mathbf{0}^{n}, \mathbf{g}^{o}\right)$ and $\mathbf{g}=\left(\mathbf{g}^{i}, \mathbf{0}^{m}\right)$ or, equivalently, conditions (7).

Chambers (2002) then defines the Bennet indicator (8a) by the difference between the Bennet revenue indicator $(8 \mathrm{~b})$ and Bennet cost indicator (8c):

$B P\left(\mathbf{p}_{t}, \mathbf{p}_{t+1}, \mathbf{w}_{t}, \mathbf{w}_{t+1}, \mathbf{y}_{t}, \mathbf{y}_{t+1}, \mathbf{x}_{t}, \mathbf{x}_{t+1}\right)=B R\left(\mathbf{p}_{t}, \mathbf{p}_{t+1}, \mathbf{y}_{t}, \mathbf{y}_{t+1}\right)-B C\left(\mathbf{w}_{t}, \mathbf{w}_{t+1}, \mathbf{x}_{t}, \mathbf{x}_{t+1}\right)$.

with

$$
B R\left(\mathbf{p}_{t}, \mathbf{p}_{t+1}, \mathbf{y}_{t}, \mathbf{y}_{t+1}\right)=\frac{1}{2}\left[\mathbf{p}_{t}\left(\mathbf{y}_{t+1}-\mathbf{y}_{t}\right)+\mathbf{p}_{t+1}\left(\mathbf{y}_{t+1}-\mathbf{y}_{t}\right)\right],
$$

and

$$
B C\left(\mathbf{w}_{t}, \mathbf{w}_{t+1}, \mathbf{x}_{t}, \mathbf{x}_{t+1}\right)=\frac{1}{2}\left[\mathbf{w}_{t}\left(\mathbf{x}_{t+1}-\mathbf{x}_{t}\right)+\mathbf{w}_{t+1}\left(\mathbf{x}_{t+1}-\mathbf{x}_{t}\right)\right] .
$$

Avoiding any estimation procedure, these Bennet indicators are straightforward to compute from available data. Hence, it is of practical interest to establish the conditions under which the Luenberger productivity indicator can be computed by a Bennet profit indicator.

Proposition 1 (Theorem 6 in Chambers (2002)). If firms maximize profit, and the technology directional distance function is quadratic with $\alpha_{t}^{i j}=\alpha_{t+1}^{i j}$ for all $i$ and $j$, $\beta_{t}^{i j}=\beta_{t+1}^{i j}$ for all $i$ and $j$ then

$$
L_{t, t+1}\left(\mathbf{x}_{t}, \mathbf{y}_{t}, \mathbf{x}_{t+1}, \mathbf{y}_{t+1} ; \mathbf{g}\right)=B P\left(\hat{\mathbf{p}}_{t}, \hat{\mathbf{p}}_{t+1}, \hat{\mathbf{w}}_{t}, \hat{\mathbf{w}}_{t+1}, \mathbf{y}_{t}, \mathbf{y}_{t+1}, \mathbf{x}_{t}, \mathbf{x}_{t+1}\right)
$$

where $\hat{\mathbf{p}}_{k}=\frac{\mathbf{p}_{k}}{\mathbf{p}_{k} \mathbf{g}^{o}+\mathbf{w}_{k} \mathbf{g}^{i}}$ and $\hat{\mathbf{w}}_{k}=\frac{\mathbf{w}_{k}}{\mathbf{p}_{k} \mathbf{g}^{o}+\mathbf{w}_{k} \mathbf{g}^{2}}$.

It turns out that the Bennet profit indicator is a superlative indicator for the Luenberger productivity indicator under an appropriate price normalization and when the directional distance function can be approximated by the quadratic functional form (5) with time-invariant second order coefficients. Time only affects the intercept and the slope of the frontier, but not its curvature. 
Both the translog and quadratic functional forms are "generalized quadratic functions" (Chambers, 1988, p.164). The translog functional form satisfies homogeneity and therefore is a popular choice to represent Shephard distance functions. The quadratic functional form satisfies the translation property and therefore can represent the directional distance function. Some papers have compared the performance of both parametrizations in terms of their ability to correctly estimate a production frontier. Färe et al. (2010) and Chambers et al. (2013) find that the quadratic functional form outperforms the translog functional form, while Färe and Vardanyan (2016) temper this conclusion somewhat by pointing out that the translog has a natural tendency to estimate convex frontiers. Furthermore, they claim that the translog often requires second-order regularity restrictions, limiting its flexibility, to ensure theoretical consistency. In contrast, the quadratic satisfies global regularity in most of the considered cases without such additional restrictions.

\section{Approximating the Luenberger-Hicks-Moorsteen indicator}

Briec and Kerstens (2004) define the LHM productivity indicator with base period $t$ - here with time-invariant directional vector - as the difference between the Luenberger output indicator and the Luenberger input indicator: ${ }^{4}$

$$
\begin{aligned}
& L H M_{t}\left(\mathbf{x}_{t+1}, \mathbf{y}_{t+1}, \mathbf{x}_{t}, \mathbf{y}_{t} ; \mathbf{g}\right) \\
& =\left(D_{t}\left(\mathbf{x}_{t}, \mathbf{y}_{t} ;\left(\mathbf{0}^{n}, \mathbf{g}^{o}\right)\right)-D_{t}\left(\mathbf{x}_{t}, \mathbf{y}_{t+1} ;\left(\mathbf{0}^{n}, \mathbf{g}^{o}\right)\right)\right) \\
& -\left(D_{t}\left(\mathbf{x}_{t+1}, \mathbf{y}_{t} ;\left(\mathbf{g}^{i}, \mathbf{0}^{m}\right)\right)-D_{t}\left(\mathbf{x}_{t}, \mathbf{y}_{t} ;\left(\mathbf{g}^{i}, \mathbf{0}^{m}\right)\right)\right) \\
& \equiv L O_{t}\left(\mathbf{x}_{t}, \mathbf{y}_{t}, \mathbf{y}_{t+1} ; \mathbf{g}^{o}\right)-\left[-L I_{t}\left(\mathbf{x}_{t}, \mathbf{x}_{t+1}, \mathbf{y}_{t} ; \mathbf{g}^{i}\right)\right] .
\end{aligned}
$$

The LHM productivity indicator with base period $t+1$ is:

$$
\begin{aligned}
& L H M_{t+1}\left(\mathbf{x}_{t+1}, \mathbf{y}_{t+1}, \mathbf{x}_{t}, \mathbf{y}_{t} ; \mathbf{g}\right) \\
& =\left(D_{t+1}\left(\mathbf{x}_{t+1}, \mathbf{y}_{t} ;\left(\mathbf{0}^{n}, \mathbf{g}^{o}\right)\right)-D_{t+1}\left(\mathbf{x}_{t+1}, \mathbf{y}_{t+1} ;\left(\mathbf{0}^{n}, \mathbf{g}^{o}\right)\right)\right) \\
& -\left(D_{t+1}\left(\mathbf{x}_{t+1}, \mathbf{y}_{t+1} ;\left(\mathbf{g}^{i}, \mathbf{0}^{m}\right)\right)-D_{t+1}\left(\mathbf{x}_{t}, \mathbf{y}_{t+1} ;\left(\mathbf{g}^{i}, \mathbf{0}^{m}\right)\right)\right) \\
& \equiv L O_{t+1}\left(\mathbf{x}_{t+1}, \mathbf{y}_{t+1}, \mathbf{y}_{t} ; \mathbf{g}^{o}\right)+L I_{t+1}\left(\mathbf{x}_{t}, \mathbf{x}_{t+1}, \mathbf{y}_{t+1} ; \mathbf{g}^{i}\right) .
\end{aligned}
$$

One takes an arithmetic mean of $L H M_{t}$ and $L H M_{t+1}$ to avoid an arbitrary choice of base periods:

$$
\begin{aligned}
& L H M_{t, t+1}\left(\mathbf{x}_{t}, \mathbf{y}_{t}, \mathbf{x}_{t+1}, \mathbf{y}_{t+1} ; \mathbf{g}\right) \\
& =\frac{1}{2}\left[L H M_{t}\left(\mathbf{x}_{t+1}, \mathbf{y}_{t+1}, \mathbf{x}_{t}, \mathbf{y}_{t} ; \mathbf{g}\right)+L H M_{t+1}\left(\mathbf{x}_{t+1}, \mathbf{y}_{t+1}, \mathbf{x}_{t}, \mathbf{y}_{t} ; \mathbf{g}\right)\right]
\end{aligned}
$$

\footnotetext{
${ }^{4}$ We follow Chambers (2002)' definition of the input indicator, swapping the places of Briec and Kerstens (2004)'s definition.
} 
Recently, Ang and Kerstens (2017a) show that the LHM indicator is "additively complete" and, following Diewert and Fox (2017), provide a decomposition in the usual components of technical change, technical inefficiency change and scale inefficiency change under minimal assumptions of the technology set. However, a superlative indicator for the LHM indicator is presently absent in the literature, which disallows easy computation of the LHM productivity indicator in practice. Our main result addresses this gap:

Proposition 2. If firms maximize profit and the input directional distance function with parameters $\left(a_{h}^{0}, \mathbf{a}_{h}, \mathbf{b}_{h}, \boldsymbol{\alpha}_{h}, \boldsymbol{\beta}_{h}, \boldsymbol{\gamma}_{h}\right)$ for $h=\{t, t+1\}$ and the output directional distance function with parameters $\left(\tilde{a}_{h}^{0}, \tilde{\mathbf{a}}_{h}, \tilde{\mathbf{b}}_{h}, \tilde{\boldsymbol{\alpha}}_{h}, \tilde{\boldsymbol{\beta}}_{h}, \tilde{\boldsymbol{\gamma}}_{h}\right)$ for $h=\{t, t+1\}$ are both quadratic with $\alpha_{t}^{i j}=\alpha_{t+1}^{i j}, \tilde{\alpha}_{t}^{i j}=\tilde{\alpha}_{t+1}^{i j}$ for all $i$ and $j, \beta_{t}^{i j}=\beta_{t+1}^{i j}, \tilde{\beta}_{t}^{i j}=\tilde{\beta}_{t+1}^{i j}$ for all $i$ and $j$ then

$$
\begin{aligned}
L H M_{t, t+1}\left(\mathbf{x}_{t}, \mathbf{y}_{t}, \mathbf{x}_{t+1}, \mathbf{y}_{t+1} ; \mathbf{g}\right) & =B P\left(\tilde{\mathbf{p}}_{t}, \tilde{\mathbf{p}}_{t+1}, \mathbf{w}_{t}^{*}, \mathbf{w}_{t+1}^{*}, \mathbf{y}_{t}, \mathbf{y}_{t+1}, \mathbf{x}_{t}, \mathbf{x}_{t+1}\right) \\
& =B R\left(\tilde{\mathbf{p}}_{t}, \tilde{\mathbf{p}}_{t+1}, \mathbf{y}_{t}, \mathbf{y}_{t+1}\right)-B C\left(\mathbf{w}_{t}^{*}, \mathbf{w}_{t+1}^{*}, \mathbf{x}_{t}, \mathbf{x}_{t+1}\right),
\end{aligned}
$$

where $\tilde{\mathbf{p}}_{k}=\frac{\mathbf{p}_{k}}{\mathbf{p}_{k} \mathbf{g}^{o}}$ and $\mathbf{w}_{k}^{*}=\frac{\mathbf{w}_{k}}{\mathbf{w}_{k} \mathbf{g}^{i}}$.

Proof. We can write

$$
L H M_{t, t+1}(\cdot)=\frac{1}{2}\left[L O_{t}(\cdot)+L O_{t+1}(\cdot)\right]+\frac{1}{2}\left[L I_{t}(\cdot)+L I_{t+1}(\cdot)\right] .
$$

From Theorem 4 in Chambers (2002), it follows that

$$
\frac{1}{2}\left[L O_{t}(\cdot)+L O_{t+1}(\cdot)\right]=B R\left(\tilde{\mathbf{p}}_{t}, \tilde{\mathbf{p}}_{t+1}, \mathbf{y}_{t}, \mathbf{y}_{t+1}\right)
$$

if firms maximize revenue, technology is quadratic with $\tilde{\beta}_{t}^{i j}=\tilde{\beta}_{t+1}^{i j}$ for all $i$ and $j$. From Theorem 2 in Chambers (2002), we know that

$$
\frac{1}{2}\left[L I_{t}(\cdot)+L I_{t+1}(\cdot)\right]=-B C\left(\mathbf{w}_{t}^{*}, \mathbf{w}_{t+1}^{*}, \mathbf{x}_{t}, \mathbf{x}_{t+1}\right)
$$

if firms minimize costs, technology is quadratic with $\alpha_{t}^{i j}=\alpha_{t+1}^{i j}$ for all $i$ and $j$. Simultaneous revenue maximization and cost minimization is profit maximization, which yields the desired result.

Proposition 2 assumes that both the input directional distance function and the output directional distance function can be represented by a quadratic functional form. As shown by Lemma 1, if the affine transformation condition holds, then the input (output) directional distance function is quadratic if and only if the output (input) directional distance function is quadratic. Since a quadratic input (output) directional distance function is not necessarily associated with a quadratic output (input) directional distance function, the equivalence between the LHM indicator and corresponding Bennet indicator requires more assumptions than Chambers (2002)' equivalence between the 
Luenberger indicator and corresponding Bennet indicator. A similar issue arises for the approximation of the ratio-based Hicks-Moorsteen index. The Törnqvist index approximates the Hicks-Moorsteen index when there is profit-maximizing behaviour and input and output distance functions can be represented by a translog functional form with time-invariant second-order coefficients (Diewert and Fox, 2010). Following Mizobuchi (2017), a translog input (output) distance function is not necessarily associated with a translog output (input) distance function. Mizobuchi (2017) shows that $\alpha$-returns-toscale renders this possible. $\alpha$-returns-to-scale is a less stringent condition than CRS, which is needed for the equivalence between the Malmquist index and the Törnqvist index. Analogously, the affine transformation condition from Lemma 1 associated with our equivalence between LHM and Bennet indicators is less stringent than graph translation homotheticity, which is a difference-based alternative to CRS.

The condition under which both Bennet indicators (cfr. $B P(\cdot)$ in Proposition 1 and Proposition 2) are equivalent follows immediately:

\section{Corollary 1.}

$$
B P\left(\tilde{\mathbf{p}}_{t}, \tilde{\mathbf{p}}_{t+1}, \mathbf{w}_{t}^{*}, \mathbf{w}_{t+1}^{*}, \mathbf{y}_{t}, \mathbf{y}_{t+1}, \mathbf{x}_{t}, \mathbf{x}_{t+1}\right)
$$

with $\left(\mathbf{g}^{i}, \mathbf{g}^{o}\right)=\left(\frac{\tau}{n \mathbf{w}_{k}}, \frac{\tau}{m \mathbf{p}_{k}}\right)$ and

$$
B P\left(\hat{\mathbf{p}}_{t}, \hat{\mathbf{p}}_{t+1}, \hat{\mathbf{w}}_{t}, \hat{\mathbf{w}}_{t+1}, \mathbf{y}_{t}, \mathbf{y}_{t+1}, \mathbf{x}_{t}, \mathbf{x}_{t+1}\right)
$$

with $\left(\mathbf{g}^{i}, \mathbf{g}^{o}\right)=\left(\frac{\tau}{2 n \mathbf{w}_{k}}, \frac{\tau}{2 m \mathbf{p}_{k}}\right)$ coincide for any $\tau \in \mathbb{R}$.

Proof. Both Bennet profit indicators only differ by their price normalization which is parametrized by the direction vectors. Both price normalizations coincide when:

$$
\begin{aligned}
\left(\tilde{\mathbf{p}}_{k}, \mathbf{w}_{k}^{*}\right) & =\left(\hat{\mathbf{p}}_{k}, \hat{\mathbf{w}}_{k}\right) \\
\Leftrightarrow\left(\frac{\mathbf{p}_{k}}{\mathbf{p}_{k} \mathbf{g}^{o}}, \frac{\mathbf{w}_{k}}{\mathbf{w}_{k} \mathbf{g}^{i}}\right) & =\left(\frac{\mathbf{p}_{k}}{\mathbf{p}_{k} \mathbf{g}^{o}+\mathbf{w}_{k} \mathbf{g}^{i}}, \frac{\mathbf{w}_{k}}{\mathbf{p}_{k} \mathbf{g}^{o}+\mathbf{w}_{k} \mathbf{g}^{i}}\right)
\end{aligned}
$$

or when both denominators equal some $\tau \in \mathbb{R}$. For the LHS this holds when $\left(\mathbf{g}^{i}, \mathbf{g}^{o}\right)=$ $\left(\frac{\tau}{n \mathbf{w}_{k}}, \frac{\tau}{m \mathbf{p}_{k}}\right)$ and for the RHS this holds when $\left(\mathbf{g}^{i}, \mathbf{g}^{o}\right)=\left(\frac{\tau}{2 n \mathbf{w}_{k}}, \frac{\tau}{2 m \mathbf{p}_{k}}\right)$.

Briec and Kerstens (2004, Proposition 6) show that if and only if the technology is (i) inversely translation homothetic in the direction of $\mathbf{g}$; and (ii) exhibits graph translation homotheticity in the direction of $\mathbf{g}$ at each time period, then the LHM indicator and the Luenberger output (input) indicator coincide. An equivalent condition in terms of Bennet profit indicators is the following: 
Corollary 2. If firms are profit-maximizing and $\mathbf{p}_{k} \mathbf{g}^{o}=\mathbf{w}_{k} \mathbf{g}^{i}$, then

$$
B P\left(\tilde{\mathbf{p}}_{t}, \tilde{\mathbf{p}}_{t+1}, \mathbf{w}_{t}^{*}, \mathbf{w}_{t+1}^{*}, \mathbf{y}_{t}, \mathbf{y}_{t+1}, \mathbf{x}_{t}, \mathbf{x}_{t+1}\right)
$$

and

$$
B P\left(\tilde{\mathbf{p}}_{t}, \tilde{\mathbf{p}}_{t+1}, \tilde{\mathbf{w}}_{t}, \tilde{\mathbf{w}}_{t+1}, \mathbf{y}_{t}, \mathbf{y}_{t+1}, \mathbf{x}_{t}, \mathbf{x}_{t+1}\right)
$$

(or $B P\left(\mathbf{p}_{t}^{*}, \mathbf{p}_{t+1}^{*}, \mathbf{w}_{t}^{*}, \mathbf{w}_{t+1}^{*}, \mathbf{y}_{t}, \mathbf{y}_{t+1}, \mathbf{x}_{t}, \mathbf{x}_{t+1}\right)$ ) (locally) coincide.

Proof. Trivial and follows directly from Corollary 8 in Chambers (2002).

The condition $\mathbf{p}_{k} \mathbf{g}^{o}=\mathbf{w}_{k} \mathbf{g}^{i}$ is also necessary for the profit function to be separable (Färe and Grosskopf, 2000).

Some remarks on the productivity indicators are in order here. Luenberger, LHM and Bennet indicators all employ an unweighted arithmetic average of current and future productivity. This implicitly yields an interpretation that current and future productivity should be equally weighted. One may also employ the perspective of managers and accountants by choosing a weighted average or fixing a certain base technology in line with Epure et al. (2011b). Fixing the current (future) period then leads to Laspeyres (Paasche)-type indicators. However, such a procedure does not yield exact relationships with superlative indicators. Färe and Zelenyuk (2019) recently provide two theoretical justifications for choosing an unweighted arithmetic average for the Luenberger input indicator, based on (i) the functional equations approach and (ii) the translation property and an additive version of the time reversal test. Analogous arguments can be provided for Luenberger and LHM indicators, and by implication, also their Bennet counterparts.

Computing the Luenberger indicator can be infeasible (Briec and Kerstens, 2009). No such infeasibilities can occur in the computation of the Luenberger-Hicks-Moorsteen indicator (Briec and Kerstens, 2011). Because the Bennet indicator does not require any estimation procedure, it can always be computed as long as prices and quantities are available.

\section{EMPIRICAL APPLICATION}

4.1. Data description. Employing the AMADEUS database, the empirical application focuses on an unbalanced sample of 5,018 Italian food and beverage companies (NACE rev. 1.1 code 15) for the years $1995-2007$. This sector consists of micro (staff in fulltime equivalents (FTE) from 10 to 20; 1, 789 observations), small (staff in FTE from 20 to $50 ; 1,864$ observations), medium (staff in FTE from 50 to $250 ; 1,250$ observations) and large (staff in FTE larger or equal to 250; 115 observations) firms. Except for large firms, all firm sizes are quite well represented in our sample. We distinguish one output and three inputs. The annual turnover is the output. The inputs include labor, materials and fixed assets. Price indexes of annual turnover, material and fixed assets 
are obtained using the EU KLEMS database. The wage is computed by the ratio of labor expense to labor quantity. The price indexes and wage are deflated to constant 1995 prices. The deflator is obtained from OECD (2018). Implicit quantities of material, fixed assets and annual turnover are calculated by the respective ratio of monetary value to price index. A full description of the data set can be found in Merlevede et al. (2015). Due to data unavailability of such prices, we are not able to identify firm-specific prices. Following Cox and Wohlgenant (1986), our analysis implicitly assumes that higher prices mean higher quality, and that this higher quality can be reflected by higher quantity. We follow the same data cleaning procedure as described in Verschelde et al. (2016). First, we remove observations with one or more improbable inputs (i.e., employment costs, deflated tangible fixed assets or deflated material costs less than 1000 EUR) or outputs (i.e., deflated turnover less than 1000 EUR). Second, we remove observations per sector-year whose sector-year growth rate fell outside the $[1 \%, 99 \%]$ of sector-year growth rates. This avoids extreme effects due to outliers and noise in the data. Deterministic computation of distance functions, as appears in our paper for Luenberger and LHM indicators, are sensitive to outliers. Bennet indicators are also sensitive to outliers. Extremely high or low quantities of outputs (inputs) will lead to extreme values in the output (input) indicator in the Bennet indicator. Table 1 shows the summary statistics of the eventual data set.

TABLE 1. Summary statistics for Italian food and beverage companies, $1995-2007$. Price indexes and wage are deflated to constant 1995 prices. There are 5,018 observations.

\begin{tabular}{|l|cccccc|}
\hline Statistic & Mean & St. Dev. & Min & $25 \%$ & $75 \%$ & Max \\
\hline Annual turnover price index & 0.900 & 0.041 & 0.848 & 0.869 & 0.912 & 1.000 \\
Implicit annual turnover quantity (in $€$ ) & $18,349,838$ & $54,140,328$ & $114,023.100$ & $3,011,035$ & $16,778,375$ & $1,660,189,355$ \\
Wage (in € per full-time equivalent) & 24,756 & 7,314 & 7,536 & 20,798 & 28,515 & 133,693 \\
Labor quantity (in full-time equivalents) & 55.037 & 165.163 & 10 & 16 & 54 & 6,160 \\
Material price index & 0.927 & 0.028 & 0.890 & 0.902 & 0.937 & 1.000 \\
Implicit material quantity (in $€$ ) & $11,778,903$ & $31,762,994$ & 1,270 & $1,569,180$ & $10,370,713$ & $872,412,992$ \\
Fixed asset price index & 0.958 & 0.023 & 0.923 & 0.938 & 0.978 & 1.000 \\
Implicit fixed assets quantity (in $€)$ & $4,119,113$ & $9,087,706$ & 1,114 & 710,514 & $4,306,673$ & $236,184,000$ \\
\hline
\end{tabular}

4.2. Estimation procedure. We empirically compare Luenberger and LHM indicators to their Bennet counterparts. The Luenberger- and LHM-approximating Bennet indicators can be easily computed using the definitions in Propositions 1 and 2, respectively. Computing Luenberger and LHM indicators requires the estimation of directional distance functions. In line with (5), we use a quadratic functional form that includes a time shifter and firm size dummy variables in the intercept $a_{h}^{0}$ : 


$$
\begin{aligned}
D_{t}\left(\mathbf{x}, \mathbf{y} ;\left(\mathbf{g}^{i}, \mathbf{g}^{o}\right)\right) & =a^{0}+\sum_{u=1}^{n} a^{u} x^{u}+\sum_{k=1}^{m} b^{k} y^{k}+\frac{1}{2} \sum_{u=1}^{n} \sum_{v=1}^{n} \alpha^{u v} x^{u} x^{v} \\
& +\frac{1}{2} \sum_{k=1}^{m} \sum_{l=1}^{m} \beta^{k l} y^{k} y^{l}+\sum_{u=1}^{n} \sum_{k=1}^{m} \gamma^{u k} x^{u} y^{k}+a^{\text {time }}(t-1995) \\
& +a^{\text {small }} d_{t}^{\text {small }}+a^{\text {medium }} d_{t}^{\text {medium }}+a^{\text {large }} d_{t}^{\text {large }} .
\end{aligned}
$$

The coefficients in this specification are all time-invariant and therefore satisfy the conditions from our theoretical results. Note that this specification is stricter than required by the theoretical results, because the linear terms are also time-invariant. Thus, time directly affects the frontier through the time shifter and the firm size dummy variables. We deterministically estimate (12) following the linear programming procedure of Aigner and Chu (1968): ${ }^{5}$

$$
\begin{aligned}
& \min _{\substack{e_{k} \geq 0, a^{0}, \mathbf{a}, \mathbf{b}, \boldsymbol{\alpha}, \boldsymbol{\beta}, \boldsymbol{\gamma}, a^{\text {time }}, a^{\text {small }}, a^{\text {medium }}, a^{\text {large }}}} \sum_{k=1}^{K} e_{k} \\
& \text { s.t. } e_{k}=(12) \\
& \forall k=1, \ldots, K \\
& (5 \mathrm{~b})-(5 \mathrm{e}) \\
& \partial D\left(\mathbf{x}_{k}, \mathbf{y}_{k} ;\left(\mathbf{g}^{i}, \mathbf{g}^{o}\right)\right) / \partial x_{k}^{u} \geq 0 \quad \forall k=1, \ldots, K ; \forall u=1, \ldots, n \\
& \partial D\left(\mathbf{x}_{k}, \mathbf{y}_{k} ;\left(\mathbf{g}^{i}, \mathbf{g}^{o}\right)\right) / \partial y_{k}^{v} \leq 0 \quad \forall k=1, \ldots, K ; \forall v=1, \ldots, m .
\end{aligned}
$$

The objective function and the first constraint fit the specified quadratic form to the data while minimizing inefficiency. The second constraint ensures compliance with the translation property for the considered directional vectors. The final two constraints impose strong disposability of the inputs and outputs, respectively (Chambers, 2002, D.4, page 753$)$. The lower bound restriction $e_{k} \geq 0$ ensures that $D_{t}\left(\mathbf{x}, \mathbf{y} ;\left(\mathbf{g}^{i}, \mathbf{g}^{o}\right)\right) \geq 0$ for all observations. Following Färe et al. (2005), we divide the values of the observations by the corresponding mean and use $\left(\mathrm{g}^{i}, \mathbf{g}^{o}\right)=\left(\mathbf{1}^{n}, \mathbf{1}^{m}\right)$ as the directional vector.

The Luenberger and LHM indicators are computed by estimating the directional distance functions and plugging them into equations (2) and (9)-(11), respectively. The coefficients of (12) are shown in Table A1 in the Appendix. ${ }^{6}$ As expected, technical inefficiency decreases with firm size. Should we conceptualize inefficiency as a measure of

\footnotetext{
${ }^{5} \mathrm{R}$ replication codes are provided as supplementary material.

${ }^{6}$ We check concavity of the estimated directional distance functions by verifying whether the eigenvalues of the Hessian are are all nonpositive (i.e., the Hessian must be negative semidefinite (Hudgins and Primont, 2007, Condition DD5 on p.34)). Only $D_{t}\left(\mathbf{x}, \mathbf{y} ;\left(\mathbf{1}^{n}, \mathbf{0}^{m}\right)\right)$ violates concavity because of a positive eigenvalue $1.81 \times 10^{-3}$.
} 
management capacity, we may conjecture that management capacity increases with firm size. For completeness, the Appendix also shows the histograms of inefficiency scores in Figure A1.

We test the plausibility that both the input- and output-oriented directional distance functions simultaneously have the quadratic functional form. The affine transformation condition in Lemma 1 in combination with our deterministic estimation procedure allows for a simple and direct test. Since there is no noise in this deterministic setting, one can conduct an Ordinary Least Squares (OLS) regression of $D_{t}\left(\mathbf{x}, \mathbf{y} ;\left(\mathbf{0}^{n}, \mathbf{1}^{m}\right)\right)$ on $D_{t}\left(\mathbf{x}, \mathbf{y} ;\left(\mathbf{1}^{n}, \mathbf{0}^{m}\right)\right)$. Here, the $R^{2}$ statistic indicates the plausibility of this affine transformation condition by showing how much of the variation in $D_{t}\left(\mathbf{x}, \mathbf{y} ;\left(\mathbf{0}^{n}, \mathbf{1}^{m}\right)\right)$ is explained by $\zeta D_{t}\left(\mathbf{x}, \mathbf{y} ;\left(\mathbf{1}^{n}, \mathbf{0}^{m}\right)\right)+\eta$. In fact, this is a joint test of both the affine transformation condition and the quadratic specification: a low $R^{2}$ can be caused by functional form misspecification of the directional distance function and/or failure of the affine transformation condition. To overcome this joint test problem, we therefore also estimate the directional distance functions using DEA and then perform the OLS regression to gauge the plausibility of the affine transformation condition in itself. Then, we repeat the OLS regression for the inefficiency scores obtained from the quadratic functional form estimation to see how this functional form specification impacts the test. The results are shown in Table 2. Both specifications yield a high $R^{2}$ of 0.89 and 0.86 for DEA and the quadratic specification, respectively. Imposing the quadratic specification has only a minor impact on the $R^{2}$. These results indicate a high plausibility of the affine transformation condition.

TABLE 2. Results for the affine transformation test. The first (second) column shows OLS results using inefficiency scores obtained with DEA (quadratic functional form).

\begin{tabular}{|l|cc|}
\hline & \multicolumn{2}{|c|}{ Dependent variable: } \\
\hline & \multicolumn{2}{|c|}{$D_{t}\left(\mathbf{x}, \mathbf{y} ;\left(\mathbf{0}^{n}, \mathbf{1}^{m}\right)\right)$} \\
& DEA & Quadratic \\
\hline$D_{t}\left(\mathbf{x}, \mathbf{y} ;\left(\mathbf{1}^{n}, \mathbf{0}^{m}\right)\right)$ & $1.677^{* * *}$ & $1.555^{* * *}$ \\
& $(0.008)$ & $(0.009)$ \\
Constant & $0.238^{* * *}$ & $0.227^{* * *}$ \\
& $(0.005)$ & $(0.006)$ \\
\hline Observations & 5,018 & 5,018 \\
$\mathrm{R}^{2}$ & 0.894 & 0.858 \\
Adjusted $\mathrm{R}^{2}$ & 0.894 & 0.858 \\
Residual Std. Error $(\mathrm{df}=5016)$ & 0.282 & 0.388 \\
F Statistic $(\mathrm{df}=1 ; 5016)$ & $42,156.740^{* * *}$ & $30,259.840^{* * *}$ \\
\hline Note: & ${ }^{*} \mathrm{p}<0.1 ;^{* *} \mathrm{p}<0.05 ;{ }^{* * *} \mathrm{p}<0.01$ \\
\hline
\end{tabular}


For completeness, Appendix B shows how the affine transformation condition can be imposed on (12) of the empirical application while imposing the parameter conditions of Lemma 2 and an affine transformation relation with unknown parameter $\zeta \in \mathbb{R}_{0}$ and assuming $\eta=0$. We note that a zero $\eta$ is not necessary for the affine transformation condition and thus imposes too severe restrictions. Using the test of Li et al. (2009), we find that the distributions for the restricted and unrestricted LHM estimates are not significantly different at the 0.05 level for seven out of twelve periods.

In conclusion, the affine transformation condition seems to be a reasonable assumption for this empirical application. We are thus confident that the results of our subsequent empirical tests of the equivalence between LHM and Bennet indicators are not an artifact of imposing the quadratic functional form for both input and output directional distance functions.

4.3. Results. Figure 1 shows the median LHM indicator and its superlative approximation by the Bennet indicator. The overall median LHM indicator is -0.0004 , while the overall median Bennet indicator (BPLHM) is -0.0029 . Both overall median TFP indicators thus indicate a very slight decline. The left-hand figure illustrates that the values of the median Bennet indicator tend to be more extreme than those of the median LHM indicator. The median Bennet indicator peaks at +0.0344 in $2003-2004$, while the highest value of the LHM indicator is +0.0236 in the same period. The lowest value of the median Bennet indicator is -0.0519 in $1995-1996$, while that of the median LHM indicator is -0.0249 in the same period. Nonetheless, the annual median and trend of both indicators are relatively close. One observes in the right-figure that the differences between the LHM and Bennet indicator are generally concentrated close to zero, with some extreme values.

Figure 2 shows the median Luenberger indicator and its superlative approximation by the Bennet indicator. The overall median Luenberger indicator is -0.0042 , while the overall median Bennet indicator (BPLuen) is -0.0182 . Remarkably, none of the median Bennet indicators exceed zero. In particular, the median Bennet indicator has plateaued to zero in 1996-1997, 1997-1998, 2003-2004, 2004-2005, 2005-2006 and 2006-2007. The lowest value of the median Bennet indicator is -0.0727 in $1995-1996$. The value of the median Luenberger indicator ranges from -0.0182 in $2000-2001$ to +0.0049 in $2003-2004$. The left-hand figure shows that the troughs of the median Bennet indicator are much more extreme than those of the median Luenberger indicator. In contrast to the preceding comparison of the Bennet and LHM estimates, the current Bennet estimates follow a different trend than the Luenberger estimates. The right-figure shows that the differences between the Luenberger and Bennet indicator are generally concentrated close to zero, with some few extreme values. 

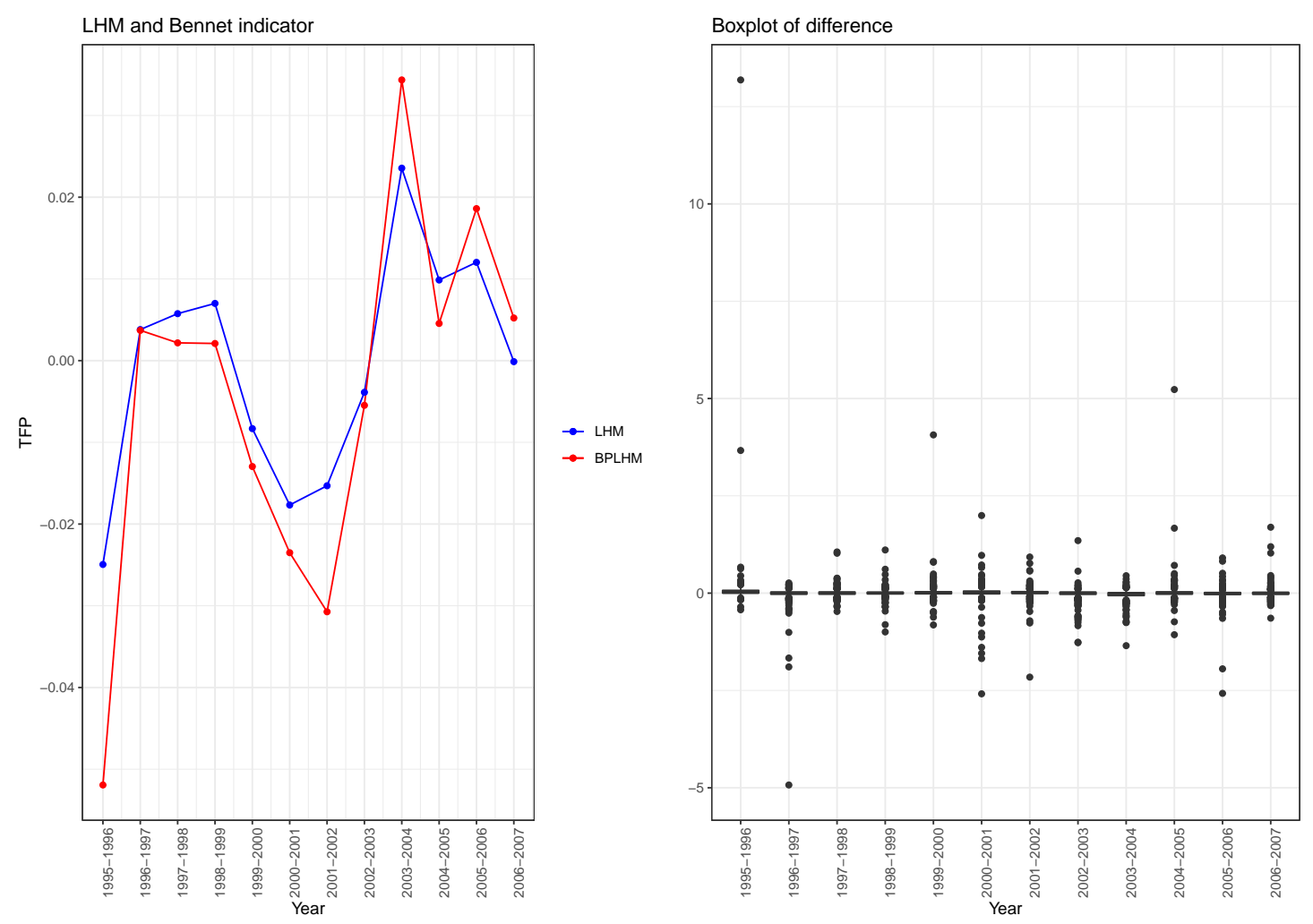

FigURE 1. Median LHM indicator and its superlative approximation by the Bennet indicator.

Figure 3 compares the Luenberger (LHM) indicator to the respective Bennet indicator in the left-hand (right-hand) scatter plot by firm size. For non-large firms, the correlation between the Luenberger indicator and respective Bennet indicator is high. The correlations are $0.83,0.78$ and 0.86 for micro, small and medium firms, respectively. For large firms, there is substantial dispersion in values. Therefore, we present the results for all large firms, on the one hand, and those for the large firms excluding observations below the 5 th and above the 95 th percentile within this size class, on the other. For the former, the correlation is 0.12 , while it is 0.52 for the latter. The correlation between the LHM indicator and Bennet indicator is even higher. The correlations are 0.98, 0.96, 0.97, 0.18 and 0.71 for micro, small, medium, large (excluding eight observations below the 5th and above the 95th percentile) and large firms (whole sample), respectively. Observe that the absolute values of Bennet estimates are generally higher than those of the corresponding Luenberger and LHM estimates, and that this especially holds for the latter. This is confirmed by Table A2 in the Appendix, which shows the median productivity estimates by firm size and per year. 

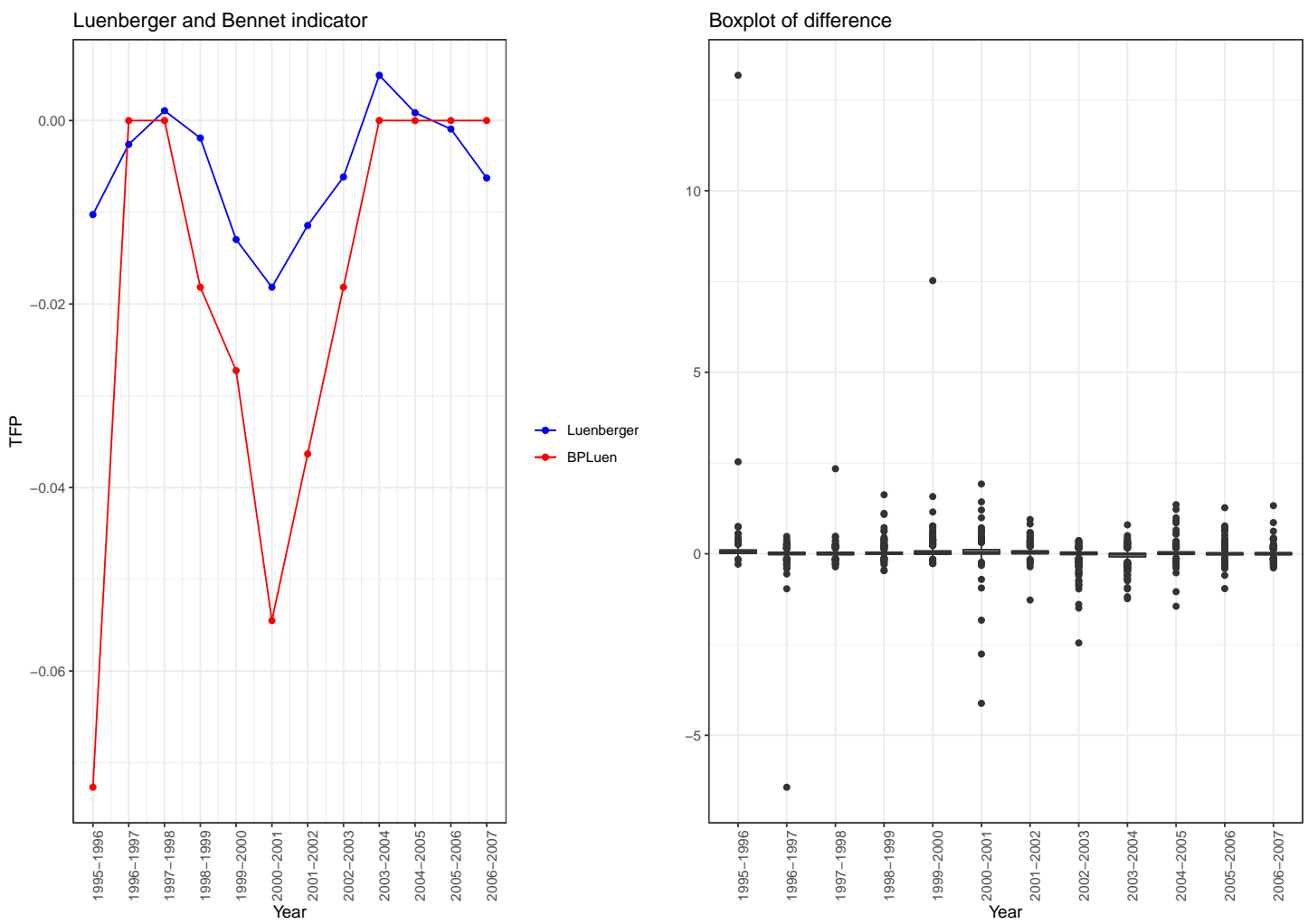

FiguRe 2. Median Luenberger indicator and its superlative approximation by the Bennet indicator.

Table 3 shows the Spearman rank correlation between the Luenberger indicator and respective Bennet indicator, on the one hand, and between the LHM indicator and respective Bennet indicator, on the other, by firm size and per year. Focusing on micro, small and medium firms, the rank correlations are generally high and significant at the 0.001 level for both comparisons throughout the whole observed period. The rank correlations between the LHM indicator and the respective Bennet indicator are consistently higher than those between the Luenberger and respective Bennet indicator. The rank correlations between LHM and Bennet indicators exceed 0.90 in all but two years (1995 - 1996 for small and medium firms). For large firms, the rank correlations fluctuate substantially, and are often not significant at the 0.001 level. This holds for both comparisons. The exception is the year $2001-2002$ which shows a very high and significant rank correlation for both indicators. These findings are in line with those obtained with the preceding correlations observed in the scatter plots. 


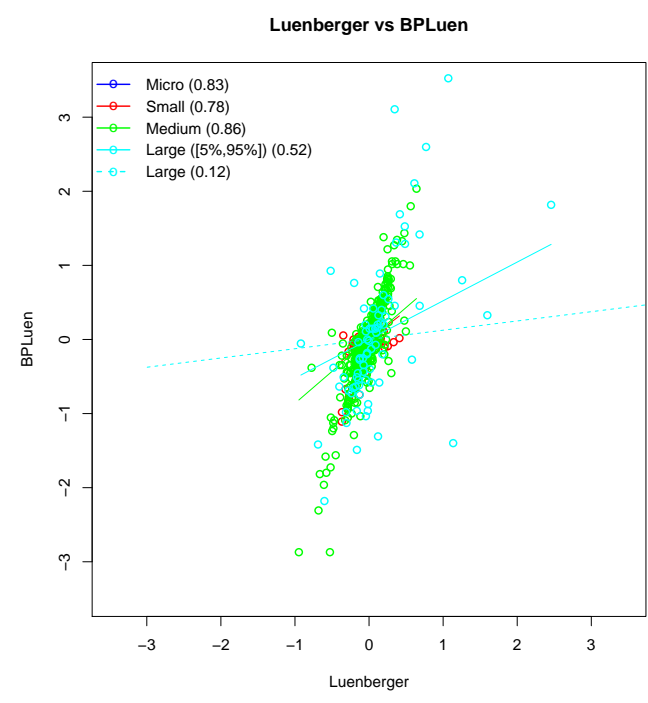

(A) Luenberger

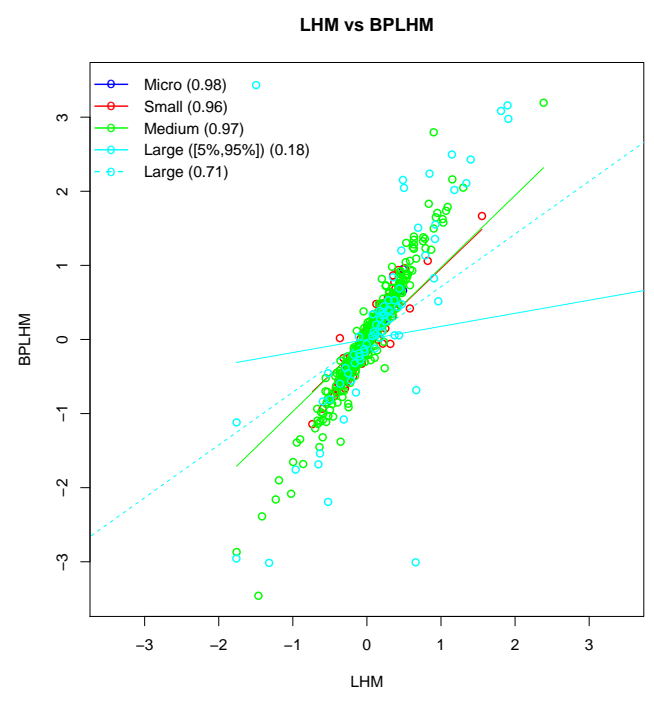

(B) LHM

FiguRE 3. Luenberger-type indicators vs. Bennet profit indicator by firm size.

Using a nonparametric test by $\mathrm{Li}$ et al. (2009), we annually compare the statistical distribution of the Luenberger (LHM) indicator to that of the respective Bennet indicator in Table 4. The null hypothesis states that the compared distributions are the same over their entire support. We reject the null hypothesis at the 0.05 level that the empirical distribution of the Luenberger indicator is equal to that of the respective Bennet indicator for all but two periods (1995- 1996 and $1997-1998)$. We also run the test by Li et al. (2009) per size class. A breakdown in size reveals that for especially small and medium firms the empirical distribution of the Luenberger indicator differs from that of its Bennet counterpart. More mixed results can be found for microfirms, where the null hypothesis cannot be rejected at the 0.05 level for $2003-2004,2004-2005$ and $2005-2006$. For large firms, no such rejection is possible for any period for which there are observations in the sample.

Focusing on the distributional comparison of LHM and Bennet indicators, we cannot reject the null hypothesis at the 0.05 level for four periods $(1998-1999,2002-2003$, $2004-2005$ and $2005-2006)$. Interestingly, we cannot reject the null hypothesis at the 0.001 level for the entire observed period. Considering the test by Li et al. (2009) per size class shows that the null hypothesis cannot be rejected for even more periods.

Overall, these results suggest that the statistical distribution of Bennet estimates is somewhat closer to that of the LHM estimates than to that of the Luenberger estimates. 
TABLE 3. Spearman rank correlation $(\rho)$ between the Luenberger/LHM indicator and respective Bennet indicator, per year and firm size.

\begin{tabular}{|c|cc|cc|cc|cc|}
\hline \multicolumn{10}{|c|}{ Luenberger and Bennet } \\
\hline Firm size & \multicolumn{2}{|c|}{ Micro } & \multicolumn{2}{c|}{ Small } & \multicolumn{2}{c|}{ Medium } & \multicolumn{2}{c|}{ Large } \\
\hline Period & $\rho$ & $p$-value & $\rho$ & $p$-value & $\rho$ & $p$-value & $\rho$ & $p$-value \\
\hline $1995-1996$ & 0.76 & 0.0000 & 0.69 & 0 & 0.59 & 0.0000 & -1 & 1 \\
$1996-1997$ & 0.78 & 0 & 0.79 & 0 & 0.81 & 0 & -1 & 0.33 \\
$1997-1998$ & 0.86 & 0 & 0.75 & 0 & 0.79 & 0 & 1 & 1 \\
$1998-1999$ & 0.89 & 0 & 0.78 & 0 & 0.86 & 0 & 0.40 & 0.75 \\
$1999-2000$ & 0.88 & 0 & 0.86 & 0 & 0.87 & 0 & 1 & 0.02 \\
$2000-2001$ & 0.89 & 0 & 0.92 & 0 & 0.88 & 0 & 0.68 & 0.05 \\
$2001-2002$ & 0.84 & 0 & 0.88 & 0 & 0.73 & 0 & 0.96 & 0 \\
$2002-2003$ & 0.90 & 0 & 0.94 & 0 & 0.94 & 0 & 0.87 & 0.001 \\
$2003-2004$ & 0.91 & 0 & 0.85 & 0 & 0.93 & 0 & 0.71 & 0.09 \\
$2004-2005$ & 0.61 & 0.0000 & 0.77 & 0 & 0.81 & 0 & 0.12 & 0.78 \\
$2005-2006$ & 0.81 & 0 & 0.73 & 0 & 0.75 & 0 & 0.79 & 0.01 \\
$2006-2007$ & 0.85 & 0 & 0.83 & 0 & 0.81 & 0 & 0.64 & 0.10 \\
\hline \multicolumn{8}{|c|}{ LHM and Bennet } \\
\hline Firm size & \multicolumn{10}{|c|}{ Micro } & \multicolumn{2}{|c|}{ Small } & \multicolumn{2}{c|}{ Medium } & \multicolumn{2}{|c|}{ Large } \\
\hline Period & $\rho$ & $p$-value & $\rho$ & $p$-value & $\rho$ & $p$-value & $\rho$ & $p$-value \\
\hline $1995-1996$ & 0.97 & 0 & 0.89 & 0 & 0.80 & 0 & 1 & 1 \\
$1996-1997$ & 0.93 & 0 & 0.95 & 0 & 0.92 & 0 & -0.50 & 1 \\
$1997-1998$ & 0.97 & 0 & 0.94 & 0 & 0.94 & 0 & 1 & 1 \\
$1998-1999$ & 0.98 & 0 & 0.94 & 0 & 0.98 & 0 & 0.80 & 0.33 \\
$1999-2000$ & 0.97 & 0 & 0.97 & 0 & 0.97 & 0 & 1 & 0.02 \\
$2000-2001$ & 0.96 & 0 & 0.98 & 0 & 0.98 & 0 & 0.95 & 0.0004 \\
$2001-2002$ & 0.97 & 0 & 0.96 & 0 & 0.94 & 0 & 0.95 & 0 \\
$2002-2003$ & 0.97 & 0 & 0.98 & 0 & 0.99 & 0 & 0.85 & 0.002 \\
$2003-2004$ & 0.95 & 0 & 0.99 & 0 & 0.98 & 0 & 1 & 0.0004 \\
$2004-2005$ & 0.94 & 0 & 0.98 & 0 & 0.91 & 0 & 0.95 & 0.0004 \\
$2005-2006$ & 0.98 & 0 & 0.98 & 0 & 0.99 & 0 & 0.98 & 0 \\
$2006-2007$ & 0.98 & 0 & 0.98 & 0 & 0.97 & 0 & 0.90 & 0.005 \\
\hline
\end{tabular}

However, we should still be careful in this assessment, as this closeness is relatively ambiguous.

So far, we have not imposed the affine transformation on the quadratic input and output directional distance functions in the LHM indicator. For completeness, we show in Appendix B how the affine transformation can be imposed empirically. The Spearman rank correlation between the LHM indicator and its Bennet counterpart is still high, but lower than in Table 3. Following the test by Li et al. (2009), we reject the null hypothesis at the 0.05 level that the empirical distribution of the (adjusted) LHM indicator is equal 
TABLE 4. Results of nonparametric density test comparing overall distributions of the Luenberger indicators and Bennet indicators. The test has been conducted on the overall sample and on subsamples by firm size.

\begin{tabular}{|c|c|c|c|c|c|c|c|c|c|c|}
\hline \multicolumn{11}{|c|}{$H_{0}:$ Luen $=$ BPLuen } \\
\hline Firm size & \multicolumn{2}{|c|}{ Overall } & \multicolumn{2}{|c|}{ Micro } & \multicolumn{2}{|c|}{ Small } & \multicolumn{2}{|c|}{ Medium } & \multicolumn{2}{|c|}{ Large } \\
\hline Period & $T_{n}$ & $p$-value & $T_{n}$ & $p$-value & $T_{n}$ & $p$-value & $T_{n}$ & $p$-value & $T_{n}$ & $p$-value \\
\hline $1995-1996$ & 45.828 & 0.920 & 6.038 & 0 & 6.535 & 0 & 3.761 & 0.005 & & \\
\hline 1996-1997 & 3.278 & 0 & 1.324 & 0.010 & 2.591 & 0.008 & 0.927 & 0.015 & & \\
\hline 1997-1998 & 14.383 & 0.333 & -1.986 & 0.045 & 1.004 & 0.015 & -5.808 & 0.015 & & \\
\hline 1998-1999 & -6.958 & 0 & 0.616 & 0 & 3.263 & 0 & -3.941 & 0.048 & & \\
\hline 1999-2000 & 1.513 & 0 & 0.873 & 0.010 & 0.051 & 0 & 2.866 & 0.003 & & \\
\hline 2000-2001 & -15.574 & 0 & 4.254 & 0 & 4.581 & 0 & 0.051 & 0.005 & 0.344 & 0.163 \\
\hline 2001-2002 & 2.937 & 0 & 4.102 & 0 & 15.290 & 0 & 6.845 & 0 & -0.227 & 0.203 \\
\hline $2002-2003$ & -8.956 & 0 & 8.557 & 0 & 7.794 & 0 & 6.985 & 0 & -0.576 & 0.376 \\
\hline 2003-2004 & -2.671 & 0 & -7.337 & 0.441 & 0.993 & 0.008 & 6.609 & 0 & 1.973 & 0.063 \\
\hline 2004-2005 & -6.521 & 0 & -4.909 & 0.093 & -3.194 & 0.003 & 1.837 & 0.003 & -0.984 & 0.534 \\
\hline 2005-2006 & 0.513 & 0 & -2.941 & 0.070 & -1.872 & 0.003 & -0.406 & 0 & & \\
\hline 2006-2007 & -10.118 & 0 & 3.517 & 0 & 0.522 & 0.030 & 3.400 & 0 & 0.406 & 0.170 \\
\hline \multicolumn{11}{|c|}{$H_{0}: \mathrm{LHM}=\mathrm{BPLHM}$} \\
\hline Firm size & \multicolumn{2}{|c|}{ Overall } & \multicolumn{2}{|c|}{ Micro } & \multicolumn{2}{|c|}{ Small } & \multicolumn{2}{|c|}{ Medium } & \multicolumn{2}{|c|}{ Large } \\
\hline $1995-1996$ & 0.010 & 0.003 & 0.487 & 0.148 & 0.812 & 0.030 & 0.576 & 0.038 & & \\
\hline 1996-1997 & 0.108 & 0.018 & -0.848 & 0.113 & -1.579 & 0.100 & -1.761 & 0.040 & & \\
\hline 1997-1998 & -9.476 & 0.005 & 0.059 & 0.190 & -2.235 & 0.060 & -2.773 & 0.058 & & \\
\hline 1998-1999 & -14.100 & 0.163 & -7.692 & 0.185 & -1.383 & 0.180 & -4.126 & 0.298 & & \\
\hline 1999-2000 & -3.506 & 0.010 & -0.396 & 0.612 & -4.350 & 0.053 & -1.281 & 0.206 & & \\
\hline 2000-2001 & -10.977 & 0.033 & -1.380 & 0.053 & 0.765 & 0.010 & -2.877 & 0.068 & -0.965 & 0.794 \\
\hline 2001-2002 & -27.840 & 0.030 & -1.464 & 0.048 & 0.465 & 0.015 & -2.809 & 0.028 & -1.402 & 0.581 \\
\hline 2002-2003 & 22.989 & 0.980 & -0.109 & 0.013 & 1.549 & 0.050 & -0.625 & 0.073 & -0.449 & 0.704 \\
\hline 2003-2004 & -4.999 & 0.013 & -2.680 & 0.143 & -0.399 & 0.015 & 1.397 & 0.020 & -1.384 & 0.684 \\
\hline 2004-2005 & -35.838 & 0.178 & -2.179 & 0.356 & -3.366 & 0.035 & -2.572 & 0.143 & -1.583 & 0.697 \\
\hline 2005-2006 & -17.595 & 0.165 & -1.684 & 0.080 & -3.922 & 0.065 & -7.870 & 0.150 & & \\
\hline 2006-2007 & 2.646 & 0.048 & -0.686 & 0.040 & -1.143 & 0.165 & 0.280 & 0.053 & -0.746 & 0.840 \\
\hline
\end{tabular}

to that of the respective Bennet indicator for all but one period $(2002-2003)$. The difference in distribution is driven by micro, small and medium firms. This contrasts with the results of Table 4 where the empirical distributions are closer. These results call for a cautious interpretation of our findings described above. Nonetheless, the empirical imposition of the affine transformation condition may be too strict, as we assume that the intercept of the input and output directional distance function is the same. Moreover, our empirical affine transformation test using OLS suggests that the affine transformation condition is a reasonable assumption for the estimated input and output directional distance functions as it can explain $89.4 \%$ of the total variation in the estimated input and output directional distance functions.

Finally, Figure 4 compares the difference between the annual change in (Profits/Fixed Assets) and the respective Bennet indicators by year. This annual change is computed by PROFITF $A_{i, t+1}-$ PROFITF $A_{i, t}$ where PROFITF $A_{i, k}=$ (Profit of firm $i$ in year $k /$ Cost of fixed assets of firm $i$ in year $k$ ). Clearly, there is a large difference between the (Profits/Fixed Assets) change and the Bennet indicators. From this figure it is clearly 
visible that the (Profits/Fixed Assets) change overestimates the actual productivity in a large majority of observations. The scatterplot in Figure 5 further shows that the annual change in (Profit/Fixed Assets) and the Bennet indicators differ significantly in all years.

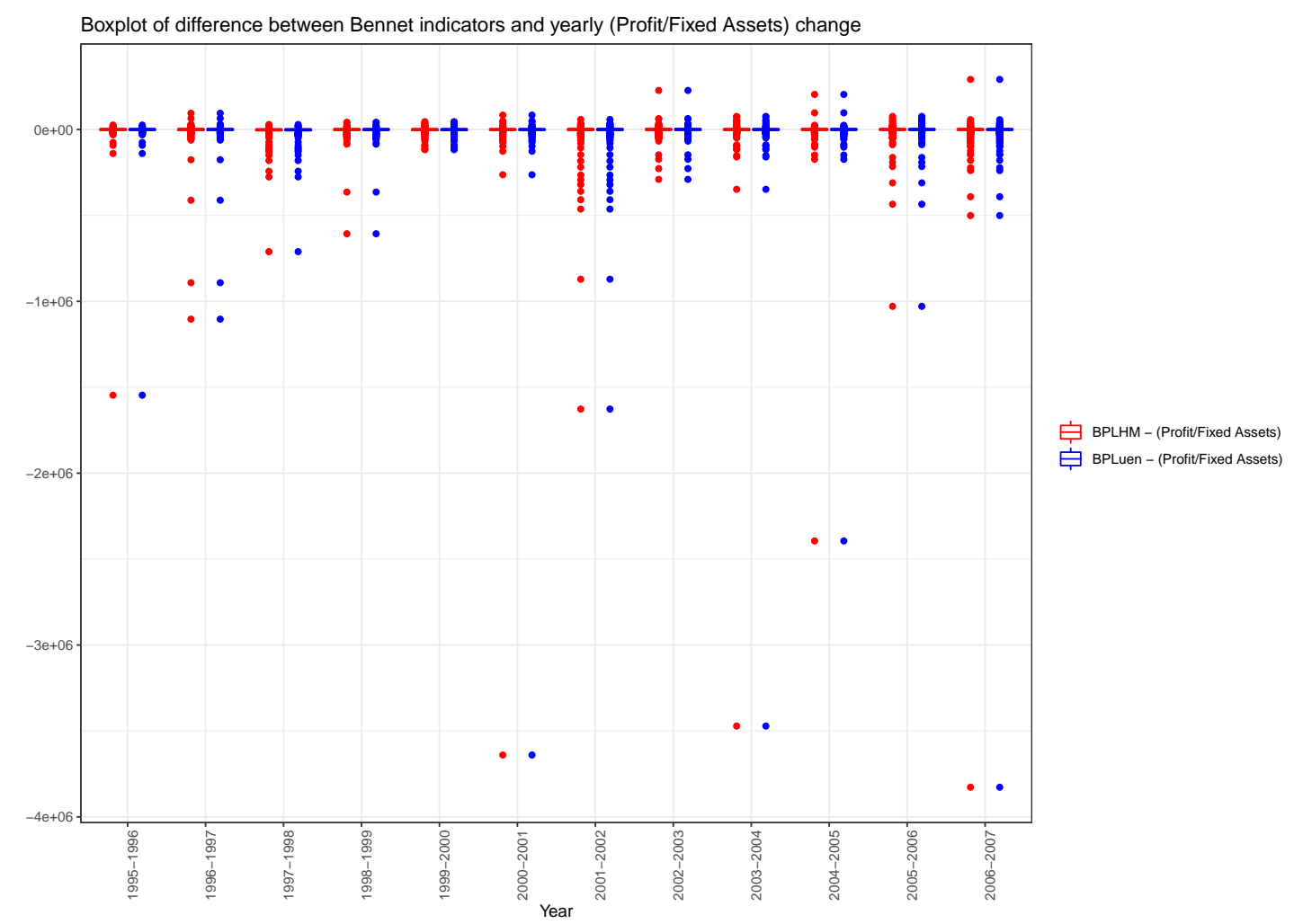

FiguRe 4. Boxplot of the difference between annual (Profit/Fixed Assets) change and the respective Bennet indicators per year.

\section{Conclusions}

This paper shows that the Bennet profit indicator is a superlative indicator for the LHM productivity indicator when one can assume that there is profit-maximizing behavior and the input and output directional distance functions are quadratic in inputs and outputs with time-invariant second-order coefficients. The Bennet profit indicator thus approximates both Chambers (2002)' Luenberger indicator and Briec and Kerstens (2004)' LHM indicator. This parallels Mizobuchi (2017)'s finding that the Törnqvist index is a superlative approximation of both Malmquist and Hicks-Moorsteen indexes under equivalent conditions. Our finding differs subtly in that our equivalence requires a different price normalization, which is not required for Mizobuchi (2017)'s finding. This 


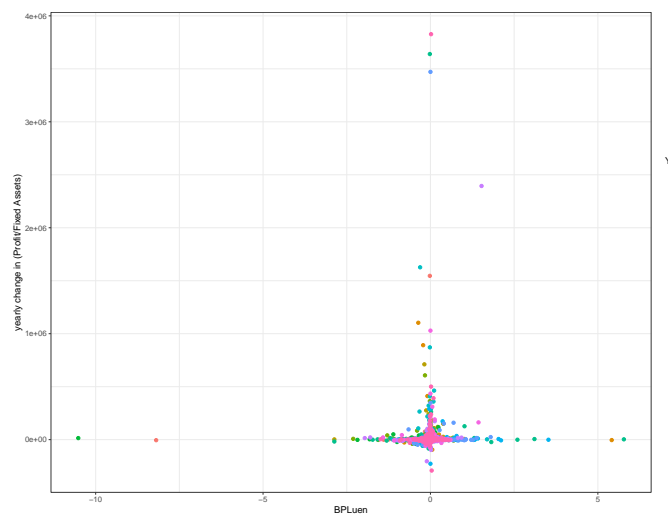

(A) BPLuen

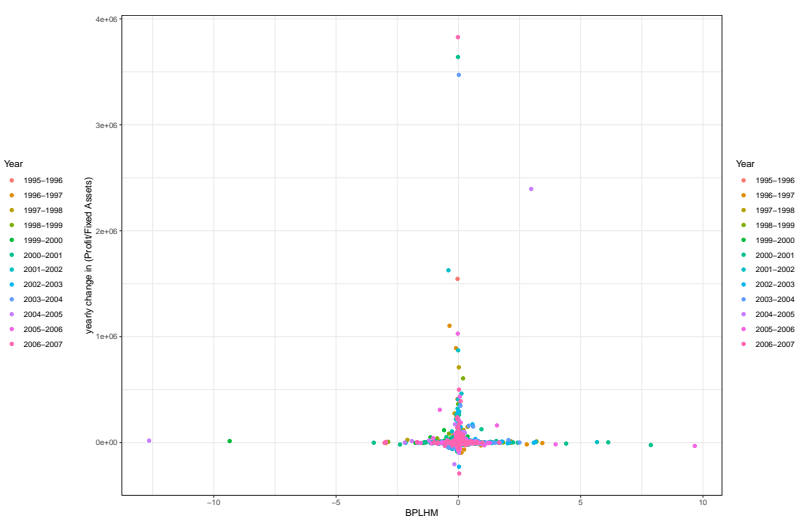

(B) BPLHM

Figure 5. Scatterplot of the Bennet indicators and the annual (Profit/Fixed Assets) change.

is a direct consequence of the fact that ratio-based indexes, unlike difference-based indicators, are unit-invariant. We also show that the Luenberger- and LHM-approximating Bennet indicators coincide for an appropriate choice of directional vectors.

The empirical application focuses on Italian food and beverage companies for the years 1995 - 2007. It shows that the Bennet estimates are similar to the LHM estimates in terms of value and (Spearman rank) correlation, and relatively close in terms of statistical distribution. This only holds to a lesser extent for the Bennet estimates approximating the Luenberger estimates. For our application, the Bennet indicator is thus a computationally simple yet empirically comparable alternative to the LHM indicator, but less so to the Luenberger indicator.

We have several recommendations for future research. First, components of productivity growth can be compared among the Luenberger and LHM indicators and their Bennet counterparts. Epure et al. (2011a) and Ang and Kerstens (2017a) respectively show how Luenberger and LHM indicators can be decomposed into technical change, technical efficiency change and scale efficiency change. Ang (2019) develops a general framework to decompose all Bennet-type indicators, including the Bennet indicator, into technical change, technical efficiency change, scale efficiency change and mix efficiency change. Technical efficiency is a measure of management capacity, which is directly related to the actual management practices. By analyzing technical efficiency change, one can thus track the change of management practices over time. Second, one could investigate which theoretical violations may deter superlative approximation. One way forward is a behavioral test of profit maximization along the lines of Varian (1984) and an in-depth analysis of the functional form and first- and second-order conditions. Third, 
we recommend to further search for superlative indicators for difference-based productivity indicators. While the literature on ratio-based approximations is rich, there seem to be only very few studies focusing on difference-based approximations. Fourth, one could extend this framework to the dynamic context. The current framework is static in that it assumes that the level of inputs and outputs can be changed instantaneously to their optimum. It ignores the sluggish adjustment of quasi-fixed inputs and the intertemporal links between production periods. From this perspective, there are several interesting developments in the literature. Following Silva and Stefanou (2003), Ang and Oude Lansink (2018) show how to appropriately account for adjustment costs in the technology in a context of dynamic profit maximization. Similar in spirit to network DEA models (see Kao (2014) for a review), Cherchye et al. (2018) introduce a nonparametric framework for intertemporal cost minimization where intertemporal production links are modeled using durable and storable inputs. Fifth, one can test the effect of management practices on productivity in line with Triebs and Kumbhakar $(2013,2018)$ in a Stochastic Frontier Analysis context, by including the relevant management variables in the quadratic functional form for the Luenberger and Luenberger-Hicks-Moorsteen indicators. Finally, it could be worthwhile to further explore the theoretical properties of the affine transformation condition. Although this paper has linked the input directional distance function to the output directional distance function using the affine transformation condition, we have remained silent on the analogous properties in the technology set. This is left for future research.

\section{REFERENCES}

Aigner, D. J. And S.-F. Chu (1968): "On estimating the industry production function," American Economic Review, 826-839.

ANG, F. (2019): "Analyzing components of productivity growth using the Bennet-Lowe indicator: An application to Welsh sheep farms," American Journal of Agricultural Economics, 101, 1262-1276.

Ang, F. And P. J. Kerstens (2017a): "Decomposing the Luenberger-HicksMoorsteen total factor productivity indicator: An application to US agriculture," European Journal of Operational Research, 260, 359-375.

— (2017b): "Exact and superlative measurement of the Luenberger-HicksMoorsteen productivity indicator," Discussion paper DPS17.02, KU Leuven, Department of Economics.

Ang, F. And A. Oude Lansink (2018): "Decomposing dynamic profit inefficiency of Belgian dairy farms," European Review of Agricultural Economics, 45, 81-99. 
Balk, B. M. (1998): Industrial Price, Quantity, and Productivity Indices: The MicroEconomic Theory and an Application, Springer US.

Balk, B. M., R. Färe, And S. Grosskopf (2003): "The theory of economic price and quantity indicators," Economic Theory, 23, 149-164.

BJurek, H. (1996): "The Malmquist Total Factor Productivity index," Scandinavian Journal of Economics, 303-313.

Briec, W., R. G. Chambers, R. Färe, and N. Peypoch (2006): "Parallel neutrality," Journal of Economics, 88, 285-305.

Briec, W. And K. Kerstens (2004): "A Luenberger-Hicks-Moorsteen productivity indicator: Its relation to the Hicks-Moorsteen productivity index and the Luenberger productivity indicator," Economic Theory, 23, 925-939.

(2009): "Infeasibility and directional distance functions with application to the determinateness of the Luenberger productivity indicator," Journal of Optimization Theory and Applications, 141, 55 .

(2011): "The Hicks-Moorsteen productivity index satisfies the determinateness axiom," The Manchester School, 79, 765-775.

Caves, D. W., L. R. Christensen, and W. E. Diewert (1982): "The economic theory of index numbers and the measurement of input, output, and productivity," Econometrica, 1393-1414.

Chambers, R. G. (1988): Applied production analysis: a dual approach, Cambridge University Press.

(1996): "A New Look at Exact Input, Output, Productivity, and Technical Change Measurement," Working Papers 197840, University of Maryland, Department of Agricultural and Resource Economics.

(2002): "Exact nonradial input, output, and productivity measurement," Economic Theory, 20, 751-765.

Chambers, R. G., R. FÄre, and S. Grosskopf (1996): "Productivity growth in APEC countries," Pacific Economic Review, 1, 181-190.

Chambers, R. G., R. Färe, S. Grosskopf, and M. Vardanyan (2013): "Generalized quadratic revenue functions," Journal of Econometrics, 173, 11-21.

Cherchye, L., B. De Rock, and P. J. Kerstens (2018): "Production with storable and durable inputs: nonparametric analysis of intertemporal efficiency," European Journal of Operational Research, 270, 498-513.

Cox, T. L. and M. K. Wohlgenant (1986): "Prices and Quality Effects in CrossSectional Demand Analysis," American Journal of Agricultural Economics, 68, 908919. 
Diewert, W. E. (1976): "Exact and superlative index numbers," Journal of Econometrics, 4, 115-145.

(1992): "Fisher ideal output, input, and productivity indexes revisited," Journal of Productivity Analysis, 3, 211-248.

Diewert, W. E. AND K. J. Fox (2010): "Malmquist and Törnqvist productivity indexes: returns to scale and technical progress with imperfect competition," Journal of Economics, 101, 73-95.

(2017): "Decomposing productivity indexes into explanatory factors," European Journal of Operational Research, 256, 275 - 291.

Epure, M., K. Kerstens, And D. Prior (2011a): "Bank productivity and performance groups: A decomposition approach based upon the Luenberger productivity indicator," European Journal of Operational Research, 211, 630-641.

_ (2011b): "Technology-based total factor productivity and benchmarking: New proposals and an application," Omega, 39, 608-619.

Eurostat (2018): "Food and beverage services statistics - NACE Rev. 2," https://ec.europa.eu/eurostat/statistics-explained/index.php/Archive: Food_and_beverage_services_statistics_-_NACE_Rev._2\#Further_Eurostat_ information, (Accessed on 2018-10-28).

Färe, R., S. Grosskopf, D.-W. Noh, And W. Weber (2005): "Characteristics of a polluting technology: theory and practice," Journal of Econometrics, 126, 469-492.

Färe, R., C. Martins-Filho, and M. Vardanyan (2010): "On functional form representation of multi-output production technologies," Journal of Productivity Analysis, 33, 81-96.

FÄre, R. AND M. VARDANYAN (2016): "A note on parameterizing input distance functions: does the choice of a functional form matter?" Journal of Productivity Analysis, 45, 121-130.

Färe, R. AND S. Grosskopf (2000): "On separability of the profit function," Journal of optimization theory and applications, 105, 609-620.

FÄRE, R. AND V. ZELENYUK (2019): "On Luenberger input, output and productivity indicators," Economics Letters, 179, 72 - 74.

Grifell-Tatjé, E. And C. A. K. Lovell (1999): "Profits and Productivity," Management Science, 45, 1177-1193.

Hudgins, L. B. And D. Primont (2007): "Derivative properties of directional technology distance functions," in Aggregation, efficiency, and measurement, Springer, $31-43$.

KAO, C. (2014): "Network data envelopment analysis: A review," European journal of operational research, 239, 1-16. 
Kerstens, K., Z. Shen, And I. Van de Woestyne (2018): "Comparing Luenberger and Luenberger-Hicks-Moorsteen productivity indicators: How well is total factor productivity approximated?" International Journal of Production Economics, 195, 311-318.

Li, Q., E. MaAsoumi, And J. S. Racine (2009): "A nonparametric test for equality of distributions with mixed categorical and continuous data," Journal of Econometrics, 148, 186-200.

Lovell, C. (2016): "Recent developments in productivity analysis," Pacific Economic Review, 21, 417-444.

Merlevede, B., M. De Zwaan, K. Lenaerts, V. Purice, et al. (2015): "Multinational networks, domestic, and foreign firms in europe," Tech. rep., Ghent University, Faculty of Economics and Business Administration.

Mizobuchi, H. (2017): "A superlative index number formula for the Hicks-Moorsteen productivity index," Journal of Productivity Analysis, 48, 167-178.

Molinos-Senante, M., T. Gomez, G. Gemar, R. Caballero, R. Sala-Garrido, And M. Mocholi-Arce (2019): "Measuring the wastewater treatment plants productivity change: Comparison of the Luenberger and Luenberger-Hicks-Moorsteen Productivity Indicators," Journal of Cleaner Production, 229, 75-83.

OECD (2018): "OECD Statistics," https://stats.oecd.org/, (Accessed on 2018-10$30)$.

O'Donnell, C. J. (2012): "An aggregate quantity framework for measuring and decomposing productivity change," Journal of Productivity Analysis, 38, 255-272.

Sala-Garrido, R., M. Molinos-Senante, and M. Mocholi-Arce (2018): "Assessing productivity changes in water companies: a comparison of the Luenberger and Luenberger-Hicks-Moorsteen productivity indicators," Urban Water Journal, 15, 626-635.

Seufert, J. H., A. Arjomandi, And K. H. Dakpo (2017): "Evaluating airline operational performance: A Luenberger-Hicks-Moorsteen productivity indicator," Transportation Research Part E: Logistics and Transportation Review, 104, 52-68.

Shen, Z., T. Balezentis, and G. D. Ferrier (2019): "Agricultural productivity evolution in China: A generalized decomposition of the Luenberger-Hicks-Moorsteen productivity indicator," China Economic Review, 57.

Silva, E. and S. E. Stefanou (2003): "Nonparametric dynamic production analysis and the theory of cost," Journal of Productivity Analysis, 19, 5-32.

Triebs, T. P. And S. C. Kumbhakar (2013): "Productivity with general indices of management and technical change," Economics Letters, 120, 18-22. 
(2018): "Management in production: from unobserved to observed," Journal of Productivity Analysis, 49, 111-121.

VARIAN, H. R. (1984): "The nonparametric approach to production analysis," Econometrica, 579-597.

Verschelde, M., M. Dumont, G. Rayp, and B. Merlevede (2016): "Semiparametric stochastic metafrontier efficiency of European manufacturing firms," Journal of Productivity Analysis, 45, 53-69. 
Appendix A. Supplementary tables and figures

TABle A1. Coefficient estimates of the directional distance functions. Eigenvalues of the Hessian are $\left(1.15 \times 10^{-14},-1.44 \times\right.$ $\left.10^{-3},-2.43 \times 10^{-3},-5.19 \times 10^{-3}\right)$ for $D_{t}\left(\mathbf{x}, \mathbf{y} ;\left(\mathbf{1}^{n}, \mathbf{1}^{m}\right)\right), \quad(1.81 \times$ $\left.10^{-3}, 2.21 \times 10^{-13},-1.19 \times 10^{-3},-1.59 \times 10^{-2}\right)$ for $D_{t}\left(\mathbf{x}, \mathbf{y} ;\left(\mathbf{1}^{n}, \mathbf{0}^{m}\right)\right)$ and $(0,-0.004,-0.007,-0.013)$ for $D_{t}\left(\mathbf{x}, \mathbf{y} ;\left(\mathbf{0}^{n}, \mathbf{1}^{m}\right)\right)$.

\begin{tabular}{|c|ccc|}
\hline & $D_{t}\left(\mathbf{x}, \mathbf{y} ;\left(\mathbf{1}^{n}, \mathbf{1}^{m}\right)\right)$ & $D_{t}\left(\mathbf{x}, \mathbf{y} ;\left(\mathbf{1}^{n}, \mathbf{0}^{m}\right)\right)$ & $D_{t}\left(\mathbf{x}, \mathbf{y} ;\left(\mathbf{0}^{n}, \mathbf{1}^{m}\right)\right)$ \\
\hline$a_{0}$ & -0.060 & -0.113 & -0.147 \\
$a_{L}$ & 0.321 & 0.638 & 0.815 \\
$a_{M}$ & 0.349 & 0.340 & 1.145 \\
$a_{F}$ & 0.029 & 0.022 & 0.126 \\
$\alpha_{L L}$ & -0.003 & -0.003 & -0.006 \\
$\alpha_{L M}$ & 0.002 & 0.002 & -0.002 \\
$\alpha_{L F}$ & 0.000 & 0.000 & 0.002 \\
$\alpha_{M M}$ & -0.003 & -0.003 & -0.012 \\
$\alpha_{M F}$ & 0.000 & 0.000 & -0.000 \\
$\alpha_{F F}$ & -0.001 & -0.001 & -0.005 \\
$\gamma_{L Y}$ & -0.001 & -0.006 & 0 \\
$\gamma_{M Y}$ & -0.000 & 0.006 & 0 \\
$\gamma_{F Y}$ & -0.000 & -0.001 & 0 \\
$b_{Y}$ & -0.301 & -0.340 & -1 \\
$\beta_{Y Y}$ & -0.002 & -0.009 & 0 \\
$a_{\text {time }}$ & 0.005 & 0.006 & 0.017 \\
$a_{\text {small }}$ & -0.012 & -0.071 & 0.046 \\
$a_{\text {medium }}$ & -0.162 & -0.357 & -0.366 \\
$a_{\text {large }}$ & -1.115 & -2.060 & -2.701 \\
\hline
\end{tabular}




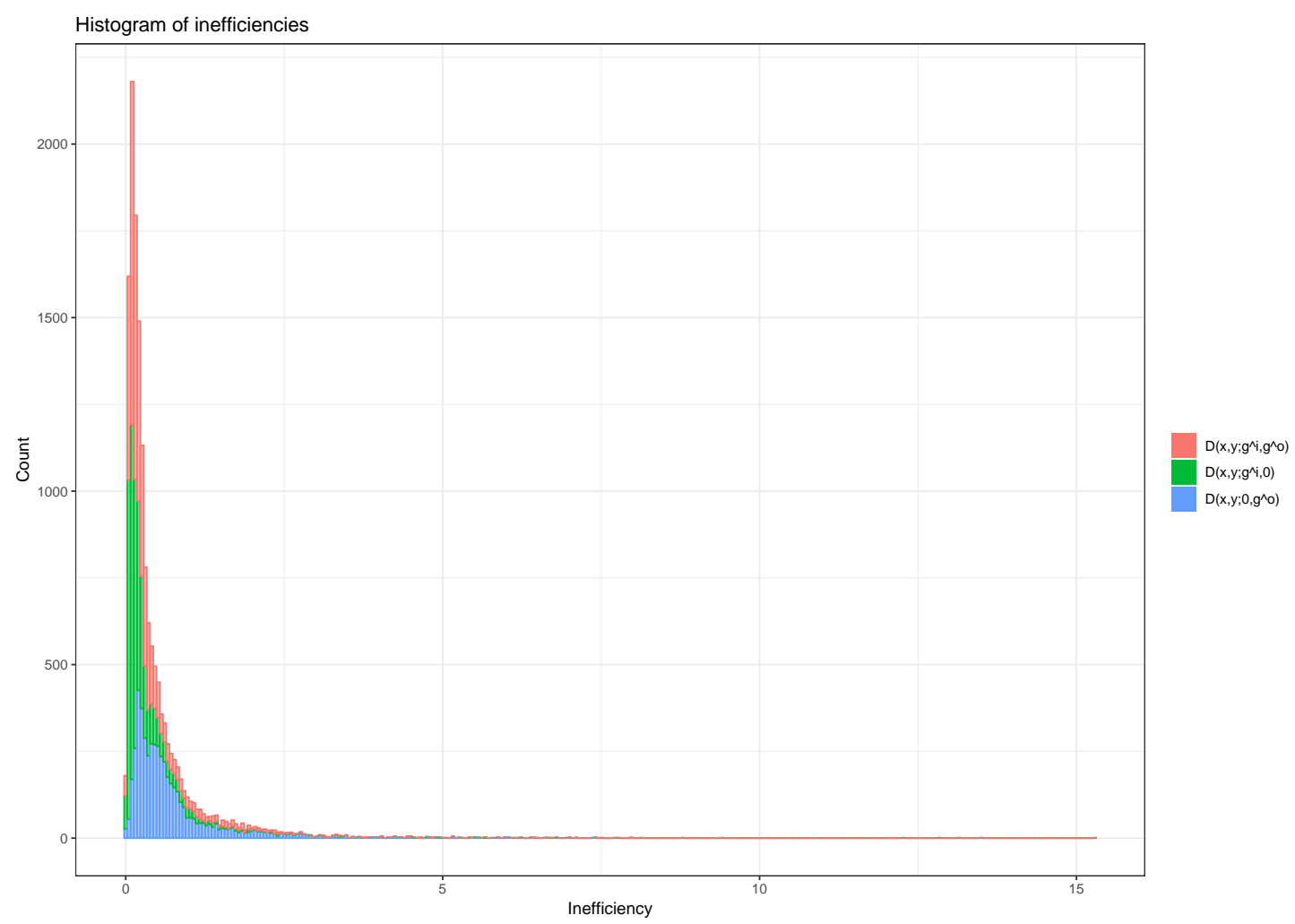

Figure A1. Histogram of inefficiency scores. 
TABle A2. Median TFP results by firm size.

\begin{tabular}{|c|cccc|cccc|}
\hline Period & \multicolumn{5}{|c|}{ Luenberger } & \multicolumn{4}{c|}{ BPLuen } \\
\hline Firm size & Micro & Small & Medium & Large & Micro & Small & Medium & Large \\
\hline $1995-1996$ & -0.008 & -0.013 & -0.015 & 3.061 & -0.036 & -0.073 & -0.127 & -4.796 \\
$1996-1997$ & -0.004 & -0.003 & 0.002 & -0.201 & -0.000 & -0.000 & -0.018 & 0.763 \\
$1997-1998$ & 0.000 & 0.004 & -0.006 & -0.043 & -0.000 & 0.000 & 0.000 & -0.236 \\
$1998-1999$ & -0.004 & 0.002 & -0.006 & 0.034 & -0.018 & -0.000 & -0.036 & -0.191 \\
$1999-2000$ & -0.004 & -0.012 & -0.055 & -0.401 & -0.000 & -0.018 & -0.145 & -0.636 \\
$2000-2001$ & -0.013 & -0.019 & -0.055 & 0.345 & -0.036 & -0.045 & -0.145 & 1.308 \\
$2001-2002$ & -0.006 & -0.011 & -0.025 & 0.105 & -0.018 & -0.036 & -0.109 & 0.227 \\
$2002-2003$ & -0.006 & -0.006 & -0.005 & 0.089 & -0.018 & -0.018 & -0.018 & 0.145 \\
$2003-2004$ & -0.000 & 0.004 & 0.049 & -0.143 & -0.000 & 0.018 & 0.164 & -0.545 \\
$2004-2005$ & 0.001 & 0.000 & 0.003 & -0.031 & 0.000 & -0.000 & -0.018 & -0.036 \\
$2005-2006$ & -0.001 & 0.002 & -0.004 & 0.045 & -0.000 & -0.000 & -0.000 & 0.109 \\
$2006-2007$ & -0.006 & -0.006 & -0.011 & -0.070 & -0.000 & -0.000 & -0.000 & -0.454 \\
\hline Period & \multicolumn{9}{|c}{ LHM } & & \multicolumn{4}{c|}{ BPLHM } & \\
\hline Firm size & Micro & Small & Medium & Large & Micro & Small & Medium & Large \\
\hline $1995-1996$ & -0.009 & -0.033 & -0.046 & 5.440 & -0.022 & -0.056 & -0.118 & -2.985 \\
$1996-1997$ & -0.002 & 0.002 & 0.038 & 0.186 & -0.002 & 0.003 & 0.031 & 2.153 \\
$1997-1998$ & 0.002 & 0.008 & 0.002 & 0.152 & -0.000 & 0.004 & -0.001 & 0.242 \\
$1998-1999$ & -0.002 & 0.009 & 0.034 & 0.341 & -0.010 & 0.008 & 0.035 & 0.431 \\
$1999-2000$ & -0.001 & -0.008 & -0.059 & 0.173 & -0.002 & -0.014 & -0.087 & 0.385 \\
$2000-2001$ & -0.009 & -0.029 & -0.062 & 0.925 & -0.017 & -0.049 & -0.137 & 2.238 \\
$2001-2002$ & -0.013 & -0.019 & -0.021 & 0.038 & -0.023 & -0.034 & -0.053 & 0.023 \\
$2002-2003$ & -0.007 & -0.005 & 0.063 & 0.320 & -0.009 & -0.007 & 0.104 & 0.288 \\
$2003-2004$ & -0.001 & 0.027 & 0.138 & 0.105 & -0.002 & 0.038 & 0.224 & -0.123 \\
$2004-2005$ & 0.007 & 0.007 & 0.023 & 0.305 & 0.006 & 0.001 & 0.001 & 0.336 \\
$2005-2006$ & 0.009 & 0.014 & 0.009 & 0.268 & 0.011 & 0.029 & 0.016 & 0.365 \\
$2006-2007$ & 0.000 & -0.003 & 0.015 & -0.386 & 0.003 & 0.001 & 0.052 & -0.521 \\
\hline
\end{tabular}




\section{Appendix B. Empirical implementation of the Lemma 2 CONDitions}

We explain in this section how one can estimate (12) of the empirical application while imposing the parameter conditions of Lemma 2 and an affine transformation relation with unknown parameters $\zeta \in \mathbb{R}_{0}$ and assuming $\eta=0$. Note that we can drop $\zeta$ in the equality constraints of Lemma 2 with 0 on the RHS. This leaves us with one constraint involving the parameter $\zeta$ : $-\zeta \sum_{u=1}^{n} a_{h}^{u} g_{u}^{i}=-1$. With an unknown $\zeta$ this constraint is vacuous and therefore can be dropped in the estimation. From this final constraint, we can then retrieve $\zeta$ after the estimation. In line with the empirical application in the main text, we deterministically estimate (12) using the modified linear program:

$$
\begin{aligned}
& \min _{\substack{e_{k} \geq 0, a^{0}, \mathbf{a}, \mathbf{b}, \boldsymbol{\alpha}, \boldsymbol{\beta}, \boldsymbol{\gamma}, a^{\text {time }}, a^{\text {small }}, a^{\text {medium }}, a^{\text {large }}}} \sum_{k=1}^{K} e_{k} \\
& \text { s.t. } e_{k}=(12) \\
& \forall k=1, \ldots, K \\
& \alpha^{u v}=\alpha^{v u}, \beta^{k l}=\beta^{l k} ; \\
& \sum_{k=1}^{m} b^{k} g_{k}^{o}=-1 \text {; } \\
& \sum_{k=1}^{m} \gamma^{u k} g_{k}^{o}=0 \\
& u=1, \ldots, n \text {; } \\
& -\sum_{v=1}^{n} \alpha^{u v} g_{v}^{i}=0, \\
& u=1, \ldots, n \text {; } \\
& \sum_{l=1}^{m} \beta^{k l} g_{l}^{o}=0, \\
& k=1, \ldots, m \text {; } \\
& -\sum_{u=1}^{n} \gamma^{u k} g_{u}^{i}=0 \\
& k=1, \ldots, m ; \\
& \partial D\left(\mathbf{x}_{k}, \mathbf{y}_{k} ;\left(\mathbf{g}^{i}, \mathbf{g}^{o}\right)\right) / \partial x_{k}^{u} \geq 0 \\
& \forall k=1, \ldots, K ; \forall u=1, \ldots, n \\
& \partial D\left(\mathbf{x}_{k}, \mathbf{y}_{k} ;\left(\mathbf{g}^{i}, \mathbf{g}^{o}\right)\right) / \partial y_{k}^{v} \leq 0 \\
& \forall k=1, \ldots, K ; \forall v=1, \ldots, m \text {. }
\end{aligned}
$$

for $\left(\mathbf{g}^{i}, \mathbf{g}^{o}\right)=\left(\mathbf{1}^{n}, \mathbf{1}^{m}\right)$. The difference with the LP in the main text is that this modified LP replaces conditions (5b)-(5e) with the conditions in Lemma 2. This yields the parameters $\boldsymbol{\theta}^{o}=\left(a^{0}, \mathbf{a}, \mathbf{b}, \boldsymbol{\alpha}, \boldsymbol{\beta}, \boldsymbol{\gamma}, a^{\text {time }}, a^{\text {small }}, a^{\text {medium }}, a^{\text {large }}\right)$ of $D_{t}\left(\mathbf{x}, \mathbf{y} ;\left(\mathbf{0}^{n}, \mathbf{1}^{m}\right)\right)$. The parameters of the input directional distance function are then retrieved from the 
affine transformation:

$$
\begin{aligned}
D_{t}\left(\mathbf{x}, \mathbf{y} ;\left(\mathbf{0}^{n}, \mathbf{1}^{m}\right)\right) & =\zeta D_{t}\left(\mathbf{x}, \mathbf{y} ;\left(\mathbf{1}^{n}, \mathbf{0}^{m}\right)\right)+\eta \\
& =\zeta D_{t}\left(\mathbf{x}, \mathbf{y} ;\left(\mathbf{1}^{n}, \mathbf{0}^{m}\right)\right),
\end{aligned}
$$

where we assume $\eta=0$ and $\zeta \in \mathbb{R}_{0}$ is unknown. Note that $\eta \neq 0$ only occurs when $D_{t}\left(\mathbf{x}, \mathbf{y} ;\left(\mathbf{0}^{n}, \mathbf{1}^{m}\right)\right)>0$ or $D_{t}\left(\mathbf{x}, \mathbf{y} ;\left(\mathbf{1}^{n}, \mathbf{0}^{m}\right)\right)>0$ which never occurs in our deterministic setting and other data envelopment techniques (such as DEA) as at least one observation is always efficient. We endogenously determine the scaling parameter $\zeta$ by setting $\zeta=$ $\sum_{u=1}^{n} a^{u} g_{u}^{i}$. The parameters of $D_{t}\left(\mathbf{x}, \mathbf{y} ;\left(\mathbf{1}^{n}, \mathbf{0}^{m}\right)\right)$ are then $\boldsymbol{\theta}^{i}=\frac{\boldsymbol{\theta}^{o}}{\zeta}$. Table A3 shows the parameter estimates. Figure A2 shows histograms comparing both input directional and output directional distance functions with and without the conditions in Lemma 2 imposed. Next, Figure A3 shows the median LHM estimates in the main text and the LHM estimates obtained with the Lemma 2 restricted directional distance functions (left) as well as boxplots of their difference in each year (right). This figure shows that both median LHM estimates follow a very similar trend over time (except in the period 2005 - 2006): the Lemma 2 restricted LHM estimates have less extreme peaks and valleys. Further, the boxplots show that the differences are generally quite small. Spearman rank correlation estimates in Table A4 further confirm that both estimates are highly similar. The nonparametric density test of Li et al. (2009) assesses whether the distributions of the LHM estimates in each year are significantly different. The first column of Table A5 indicates that their respective distributions on the entire sample are not significantly different in the majority of years: the null hypothesis $H_{0}$ cannot be rejected for 7 out of 12 periods at the 0.05 level. The test results on subsamples per firm size in the other columns reveal that the null hypothesis can only be rejected for small firms in 2 periods (i.e., $2001-2002$ and $2003-2004$ ) at the 0.05 level and in no period for the other firm sizes.

The differences between the estimated Lemma 2 restricted directional distance functions and those in the main text are at least partially explained by the fact that the affine transformation condition does not hold perfectly in this data sample: $10.6 \%$ of the variation in $D_{t}\left(\mathbf{x}, \mathbf{y} ;\left(\mathbf{0}^{n}, \mathbf{1}^{m}\right)\right)$ remains unexplained (as indicated by the $R^{2}=0.894$ statistic in Table 2). In cases where this residual variation is even smaller one expects even less difference between these directional distance functions. 
TABLE A3. Coefficient estimates of the input and output directional distance functions when the extra conditions in Lemma 2 are imposed. The parameters of the affine transformation are $\zeta=2.072$ and assuming $\eta=0$. Both directional distance functions are concave (i.e., Hessian is negative semidefinite).

\begin{tabular}{|c|cc|}
\hline & $D_{t}\left(\mathbf{x}, \mathbf{y} ;\left(\mathbf{1}^{n}, \mathbf{0}^{m}\right)\right)$ & $D_{t}\left(\mathbf{x}, \mathbf{y} ;\left(\mathbf{0}^{n}, \mathbf{1}^{m}\right)\right)$ \\
\hline$a_{0}$ & -0.071 & -0.146 \\
$a_{L}$ & 0.397 & 0.823 \\
$a_{M}$ & 0.543 & 1.126 \\
$a_{F}$ & 0.059 & 0.123 \\
$\alpha_{L L}$ & -0.008 & -0.017 \\
$\alpha_{L M}$ & 0.006 & 0.012 \\
$\alpha_{L F}$ & 0.002 & 0.005 \\
$\alpha_{M M}$ & -0.007 & -0.015 \\
$\alpha_{M F}$ & 0.001 & 0.003 \\
$\alpha_{F F}$ & -0.004 & -0.008 \\
$\gamma_{L Y}$ & 0 & 0 \\
$\gamma_{M Y}$ & 0 & 0 \\
$\gamma_{F Y}$ & 0 & 0 \\
$b_{Y}$ & -0.483 & -1 \\
$\beta_{Y Y}$ & 0 & 0 \\
$a_{\text {time }}$ & 0.008 & 0.017 \\
$a_{\text {small }}$ & 0.028 & 0.057 \\
$a_{\text {medium }}$ & -0.176 & -0.365 \\
$a_{\text {large }}$ & -1.401 & -2.903 \\
\hline
\end{tabular}



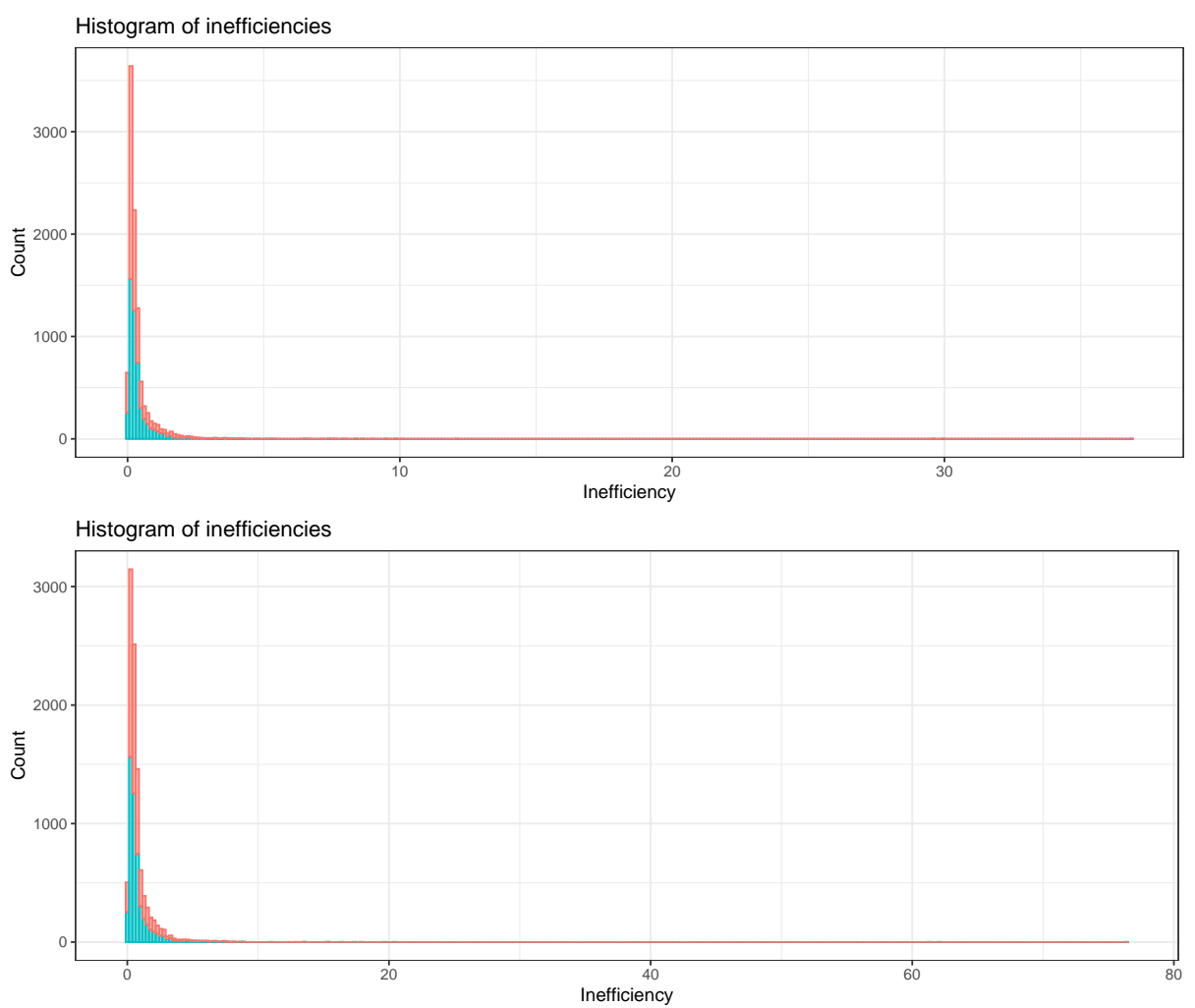

Figure A2. Histograms of inefficiency scores for $D_{t}\left(\mathbf{x}, \mathbf{y} ;\left(\mathbf{1}^{n}, \mathbf{0}^{m}\right)\right)$ and $D_{t}\left(\mathbf{x}, \mathbf{y} ;\left(\mathbf{0}^{n}, \mathbf{1}^{m}\right)\right)$ with and without the extra conditions in Lemma 2. 

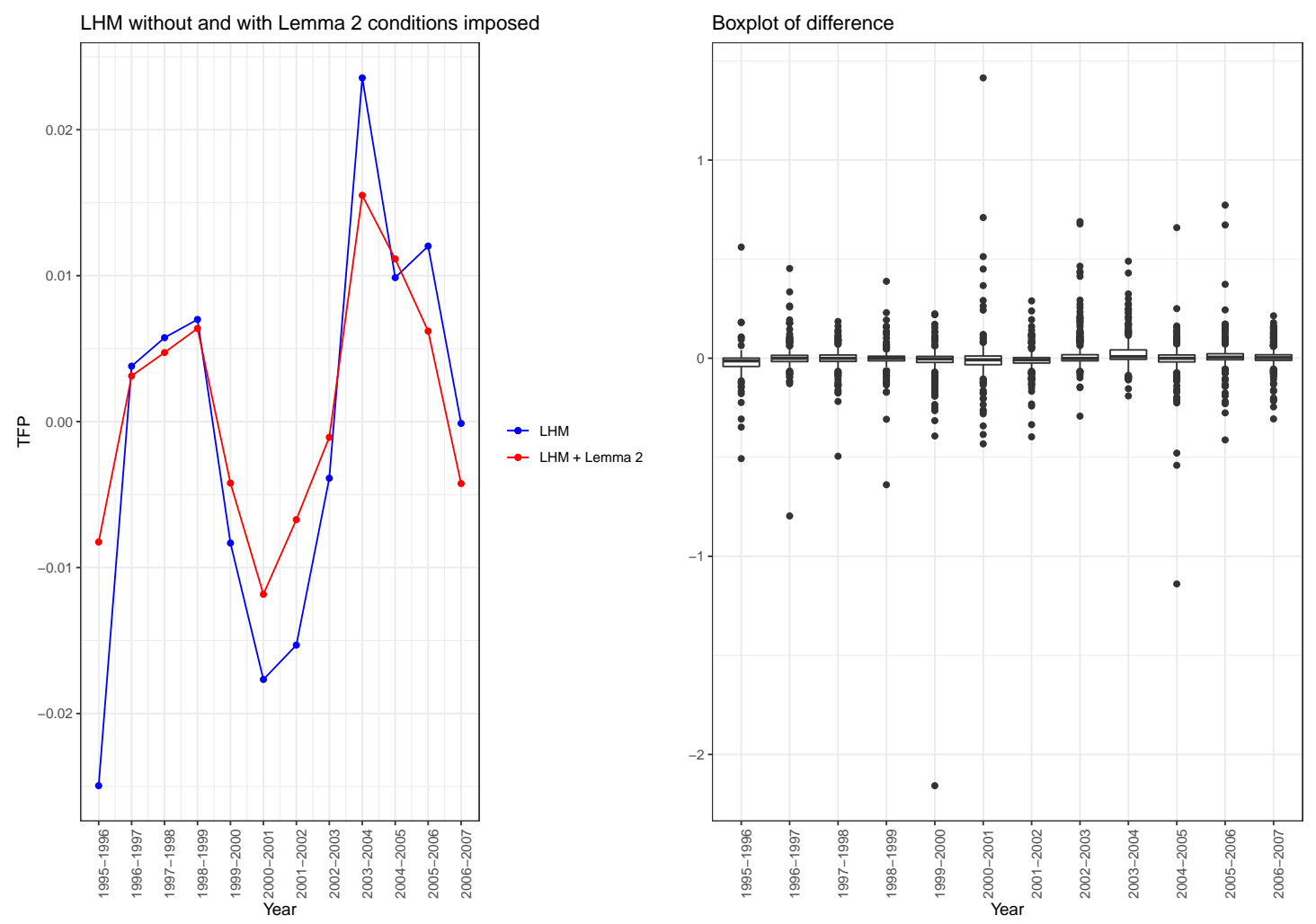

Figure A3. LHM with and without the extra conditions in Lemma 2. 
TABLE A4. Spearman rank correlation $(\rho)$ between (i) the LHM estimates with and without Lemma 2 imposed and (ii) LHM with Lemma 2 imposed and BPLHM, per year and firm size.

\begin{tabular}{|c|c|c|c|c|c|c|c|c|}
\hline \multicolumn{9}{|c|}{ LHM and LHM with Lemma 2} \\
\hline Firm size & \multicolumn{2}{|c|}{ Micro } & \multicolumn{2}{|c|}{ Small } & \multicolumn{2}{|c|}{ Medium } & \multicolumn{2}{|c|}{ Large } \\
\hline Period & $\rho$ & $p$-value & $\rho$ & $p$-value & $\rho$ & $p$-value & $\rho$ & $p$-value \\
\hline $1995-1996$ & 0.85 & 0 & 0.84 & 0 & 0.78 & 0 & 1 & 1 \\
\hline 1996-1997 & 0.96 & 0 & 0.91 & 0 & 0.93 & 0 & 1 & 0.33 \\
\hline 1997-1998 & 0.94 & 0 & 0.92 & 0 & 0.85 & 0 & 1 & 1 \\
\hline 1998-1999 & 0.95 & 0 & 0.95 & 0 & 0.98 & 0 & 0.80 & 0.33 \\
\hline 1999-2000 & 0.96 & 0 & 0.95 & 0 & 0.96 & 0 & 1 & 0.02 \\
\hline $2000-2001$ & 0.96 & 0 & 0.96 & 0 & 0.95 & 0 & 0.95 & 0.0004 \\
\hline 2001-2002 & 0.92 & 0 & 0.94 & 0 & 0.86 & 0 & 0.89 & 0.001 \\
\hline 2002-2003 & 0.91 & 0 & 0.97 & 0 & 0.98 & 0 & 0.99 & 0 \\
\hline 2003-2004 & 0.97 & 0 & 0.97 & 0 & 0.96 & 0 & 0.96 & 0.003 \\
\hline 2004-2005 & 0.91 & 0 & 0.97 & 0 & 0.94 & 0 & 0.93 & 0.001 \\
\hline 2005-2006 & 0.98 & 0 & 0.94 & 0 & 0.97 & 0 & 0.93 & 0.0001 \\
\hline $2006-2007$ & 0.96 & 0 & 0.96 & 0 & 0.96 & 0 & 1 & 0.0000 \\
\hline \multicolumn{9}{|c|}{ LHM with Lemma 2 and BPLHM } \\
\hline Firm size & \multicolumn{2}{|c|}{ Micro } & \multicolumn{2}{|c|}{ Small } & \multicolumn{2}{|c|}{ Medium } & \multicolumn{2}{|c|}{ Large } \\
\hline Period & $\rho$ & $p$-value & $\rho$ & $p$-value & $\rho$ & $p$-value & $\rho$ & $p$-value \\
\hline 1995-1996 & 0.72 & 0.0000 & 0.59 & 0.0000 & 0.39 & 0.004 & 1 & 1 \\
\hline 1996-1997 & 0.82 & 0 & 0.79 & 0 & 0.76 & 0 & -0.50 & 1 \\
\hline 1997-1998 & 0.86 & 0 & 0.80 & 0 & 0.68 & 0 & 1 & 1 \\
\hline 1998-1999 & 0.90 & 0 & 0.82 & 0 & 0.93 & 0 & 0.60 & 0.42 \\
\hline 1999-2000 & 0.88 & 0 & 0.87 & 0 & 0.89 & 0 & 1 & 0.02 \\
\hline 2000-2001 & 0.87 & 0 & 0.91 & 0 & 0.88 & 0 & 0.93 & 0.001 \\
\hline 2001-2002 & 0.83 & 0 & 0.82 & 0 & 0.70 & 0 & 0.79 & 0.01 \\
\hline 2002-2003 & 0.81 & 0 & 0.92 & 0 & 0.95 & 0 & 0.88 & 0.001 \\
\hline 2003-2004 & 0.87 & 0 & 0.93 & 0 & 0.90 & 0 & 0.96 & 0.003 \\
\hline $2004-2005$ & 0.79 & 0 & 0.92 & 0 & 0.77 & 0 & 0.82 & 0.01 \\
\hline 2005-2006 & 0.94 & 0 & 0.87 & 0 & 0.93 & 0 & 0.90 & 0.001 \\
\hline 2006-2007 & 0.90 & 0 & 0.89 & 0 & 0.87 & 0 & 0.90 & 0.005 \\
\hline
\end{tabular}


TABLE A5. Results of nonparametric density test comparing overall distributions of (i) the LHM estimates with and without additional conditions of Lemma 2 imposed and (ii) BPLHM and LHM with additional conditions of Lemma 2 imposed. The test is done on the overall sample and on subsamples by firm size.

\begin{tabular}{|c|c|c|c|c|c|c|c|c|c|c|}
\hline \multicolumn{11}{|c|}{$H_{0}:$ LHM $=$ LHM with Lemma 2} \\
\hline Firm size & \multicolumn{2}{|c|}{ Overall } & \multicolumn{2}{|c|}{ Micro } & \multicolumn{2}{|c|}{ Small } & \multicolumn{2}{|c|}{ Medium } & \multicolumn{2}{|c|}{ Large } \\
\hline Period & $T_{n}$ & $p$-value & $T_{n}$ & $p$-value & $T_{n}$ & $p$-value & $T_{n}$ & $p$-value & $T_{n}$ & $p$-value \\
\hline $1995-1996$ & -11.298 & 0.759 & 0.763 & 0.296 & -2.843 & 0.737 & -0.781 & 0.391 & & \\
\hline 1996-1997 & 44.930 & 0.038 & 1.121 & 0.188 & -3.649 & 0.674 & 3.140 & 0.256 & & \\
\hline 1997-1998 & 10.095 & 0.030 & 2.872 & 0.201 & 1.755 & 0.175 & 4.132 & 0.221 & & \\
\hline 1998-1999 & 17.849 & 0.170 & 10.713 & 0.193 & -0.681 & 0.664 & 1.029 & 0.589 & & \\
\hline 1999-2000 & 9.554 & 0.013 & 0.431 & 0.376 & 12.889 & 0.068 & 1.641 & 0.323 & & \\
\hline 2000-2001 & -8.408 & 0.912 & 3.855 & 0.175 & -1.871 & 0.301 & 0.171 & 0.581 & -1.007 & 0.962 \\
\hline 2001-2002 & -2.749 & 0.426 & 7.393 & 0.080 & 5.421 & 0.023 & 5.867 & 0.195 & -1.166 & 0.972 \\
\hline 2002-2003 & -5.219 & 0.444 & 4.856 & 0.053 & 3.851 & 0.055 & 1.060 & 0.243 & -0.559 & 0.840 \\
\hline 2003-2004 & 8.630 & 0.010 & 4.985 & 0.318 & 5.567 & 0.038 & 4.010 & 0.055 & -1.312 & 0.992 \\
\hline 2004-2005 & 120.761 & 0.088 & 2.968 & 0.529 & 4.036 & 0.093 & 5.686 & 0.298 & -0.386 & 0.910 \\
\hline 2005-2006 & 125.453 & 0.103 & 4.196 & 0.115 & 6.720 & 0.145 & 9.592 & 0.168 & & \\
\hline 2006-2007 & 24.379 & 0.030 & 0.160 & 0.286 & 4.347 & 0.228 & 1.138 & 0.556 & -1.144 & 0.995 \\
\hline \multicolumn{11}{|c|}{$H_{0}:$ BPLHM = LHM with Lemma 2} \\
\hline Firm size & \multicolumn{2}{|c|}{ Overall } & \multicolumn{2}{|c|}{ Micro } & \multicolumn{2}{|c|}{ Small } & \multicolumn{2}{|c|}{ Medium } & \multicolumn{2}{|c|}{ Large } \\
\hline Period & $T_{n}$ & $p$-value & $T_{n}$ & $p$-value & $T_{n}$ & $p$-value & $T_{n}$ & $p$-value & $T_{n}$ & $p$-value \\
\hline $1995-1996$ & 8.131 & 0 & 5.484 & 0.003 & 6.825 & 0.005 & 8.946 & 0 & & \\
\hline 1996-1997 & 55.204 & 0 & 11.382 & 0.003 & 7.646 & 0 & 16.485 & 0.010 & & \\
\hline 1997-1998 & 54.035 & 0 & 8.097 & 0.003 & 15.546 & 0 & 18.558 & 0.013 & & \\
\hline 1998-1999 & 42.640 & 0.023 & 26.640 & 0.033 & 5.209 & 0.043 & 9.278 & 0.098 & & \\
\hline 1999-2000 & 37.189 & 0 & 3.055 & 0.035 & 28.782 & 0.003 & 8.055 & 0.008 & & \\
\hline 2000-2001 & 15.776 & 0.003 & 17.086 & 0.003 & 11.735 & 0 & 16.303 & 0.023 & -0.191 & 0.604 \\
\hline 2001-2002 & 52.888 & 0.008 & 19.278 & 0.003 & 21.715 & 0 & 20.264 & 0.003 & 0.510 & 0.589 \\
\hline 2002-2003 & -35.447 & 0.922 & 18.977 & 0 & 11.669 & 0 & 12.431 & 0 & -0.061 & 0.373 \\
\hline 2003-2004 & 42.435 & 0 & 14.370 & 0.035 & 21.427 & 0 & 13.921 & 0 & 0.065 & 0.825 \\
\hline 2004-2005 & 145.407 & 0.005 & 7.154 & 0.140 & 25.571 & 0 & 14.529 & 0.015 & 1.093 & 0.566 \\
\hline 2005-2006 & 139.389 & 0.003 & 15.828 & 0.003 & 20.242 & 0.003 & 28.261 & 0.020 & & \\
\hline 2006-2007 & 30.545 & 0 & 13.783 & 0 & 9.472 & 0.005 & 8.859 & 0 & -0.806 & 0.782 \\
\hline
\end{tabular}

